August 2003 - NREL/TP-500-34632

\title{
A Demonstration of the Ability of RCAS to Model Wind Turbines
}

\author{
J. Jonkman \\ J. Cotrell
}

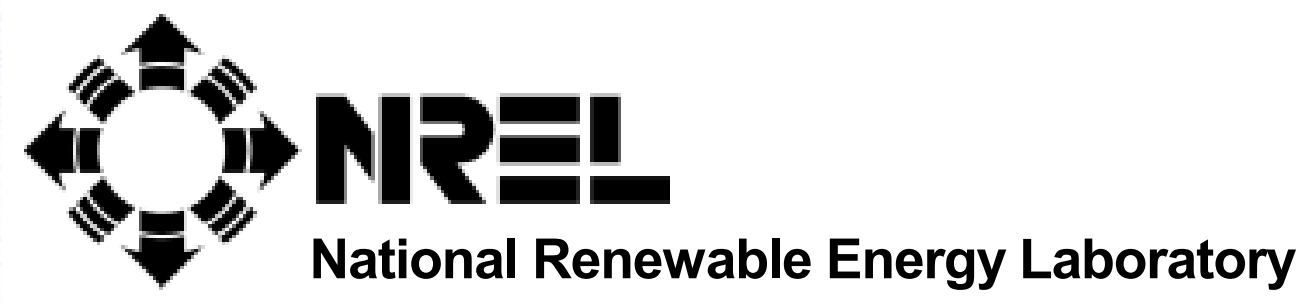

1617 Cole Boulevard

Golden, Colorado 80401-3393

NREL is a U.S. Department of Energy Laboratory

Operated by Midwest Research Institute $\bullet$ Battelle $\bullet$ Bechtel

Contract No. DE-AC36-99-G010337 


\section{A Demonstration of the Ability of RCAS to Model Wind Turbines}

\section{J. Jonkman \\ J. Cotrell}

Prepared under Task No. WER3.2050

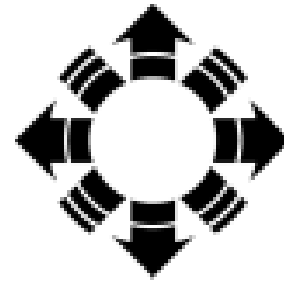

National Renewable Energy Laboratory

1617 Cole Boulevard

Golden, Colorado 80401-3393

NREL is a U.S. Department of Energy Laboratory

Operated by Midwest Research Institute • Battelle • Bechtel

Contract No. DE-AC36-99-G010337 


\section{NOTICE}

This report was prepared as an account of work sponsored by an agency of the United States government. Neither the United States government nor any agency thereof, nor any of their employees, makes any warranty, express or implied, or assumes any legal liability or responsibility for the accuracy, completeness, or usefulness of any information, apparatus, product, or process disclosed, or represents that its use would not infringe privately owned rights. Reference herein to any specific commercial product, process, or service by trade name, trademark, manufacturer, or otherwise does not necessarily constitute or imply its endorsement, recommendation, or favoring by the United States government or any agency thereof. The views and opinions of authors expressed herein do not necessarily state or reflect those of the United States government or any agency thereof.

Available electronically at http://www.osti.gov/bridge

Available for a processing fee to U.S. Department of Energy and its contractors, in paper, from:

U.S. Department of Energy

Office of Scientific and Technical Information

P.O. Box 62

Oak Ridge, TN 37831-0062

phone: 865.576 .8401

fax: 865.576.5728

email: reports@adonis.osti.gov

Available for sale to the public, in paper, from:

U.S. Department of Commerce

National Technical Information Service

5285 Port Royal Road

Springfield, VA 22161

phone: 800.553.6847

fax: 703.605.6900

email: orders@ntis.fedworld.gov

online ordering: http://www.ntis.gov/ordering.htm 


\section{Table of Contents}

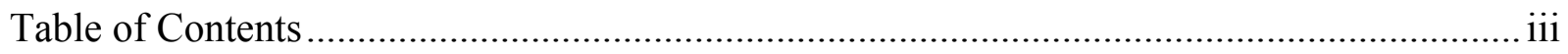

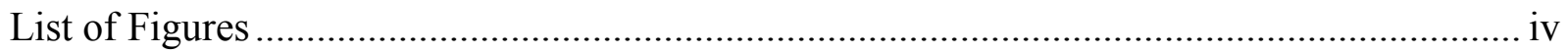

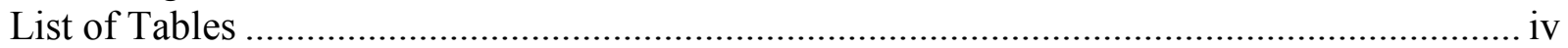

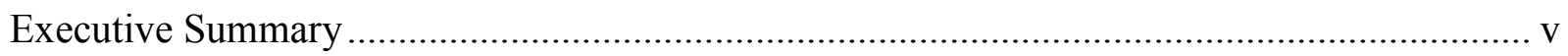

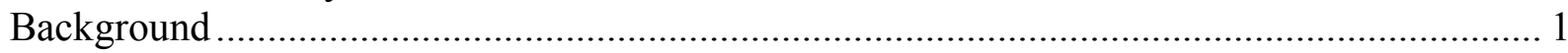

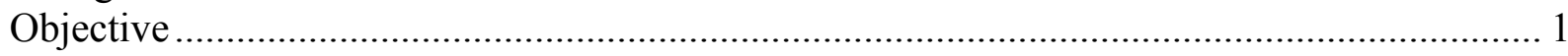

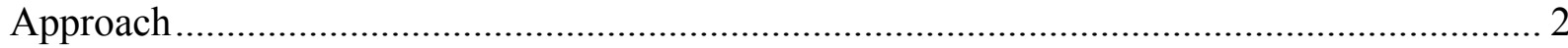

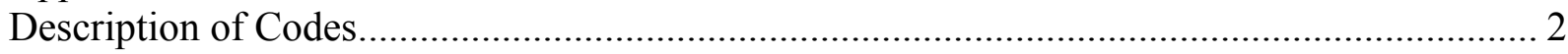

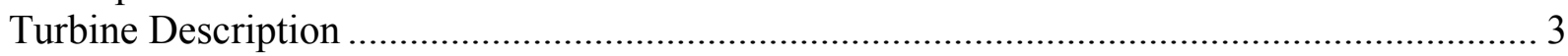

Description of Models................................................................................................ 4

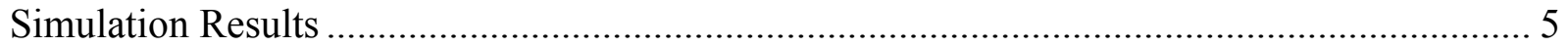

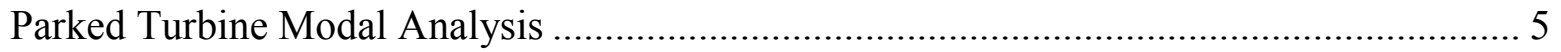

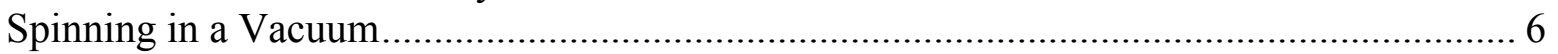

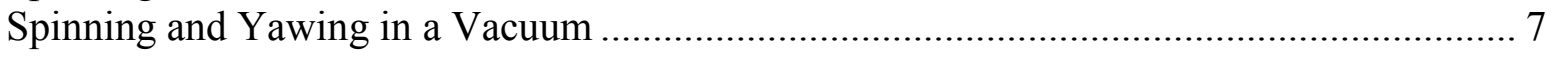

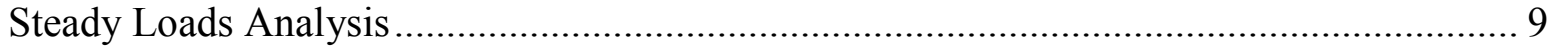

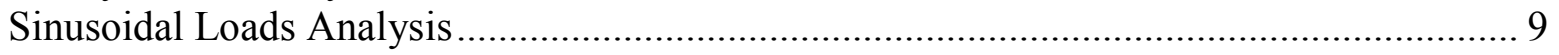

A Critique of RCAS's Usability and Applicability in the Wind Industry .............................. 9

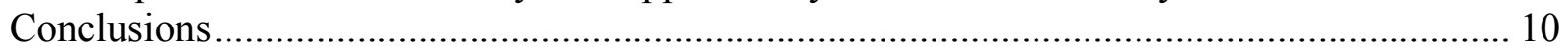

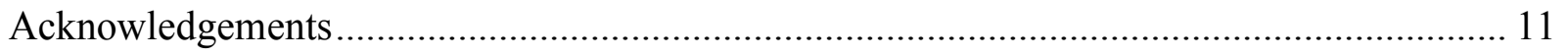

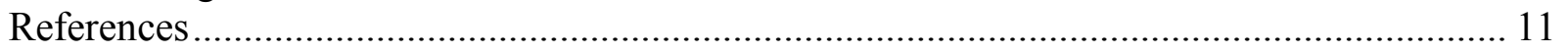

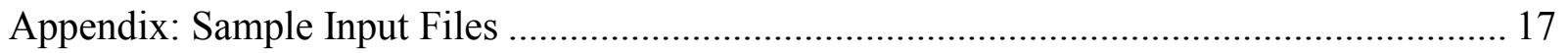

FAST Input File for the Turbine Spinning in a Vacuum .............................................. 17

FAST Blade Input File..................................................................................... 21

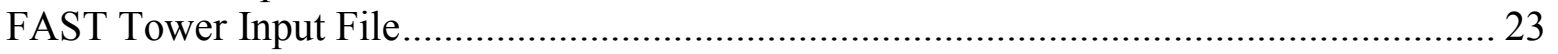

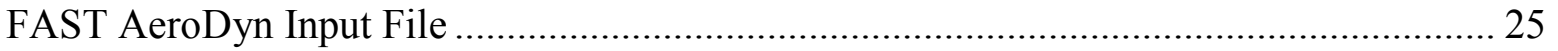

RCAS Input File for the Turbine Spinning in a Vacuum ........................................... 26

RCAS Blade Properties Input File ........................................................................... 39

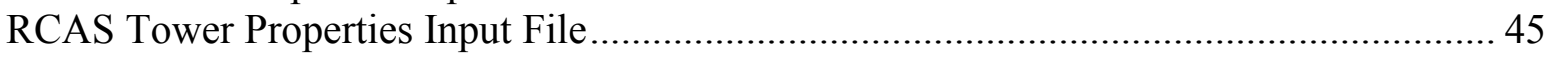

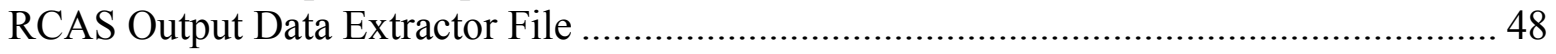




\section{List of Figures}

Figure 1: Graphical representation of analyses performed in the code demonstration................. 5

Figure 2: Radial blade root forces for the turbine spinning in a vacuum. ................................ 7

Figure 3: Output yaw angle for the turbine spinning and yawing in a vacuum analysis............. 8

Figure 4: Yaw moment for the turbine spinning and yawing in a vacuum................................ 8

Figure 5: Selected responses for the turbine spinning in a vacuum.................................... 13

Figure 6: Selected responses for the turbine spinning and yawing in a vacuum..................... 14

Figure 7: Selected responses for $12 \mathrm{~m} / \mathrm{s}$ steady loads applied to the turbine........................... 15

Figure 8: Selected responses for $12 \mathrm{~m} / \mathrm{s}$ sinusoidal loads applied to the turbine........................ 16

\section{List of Tables}

Table 1: Program Versions Used in this Study ................................................................... 2

Table 2: Summary of Baseline Turbine Design Properties .................................................... 3

Table 3: Non-Rotating Full System Natural Frequencies .................................................. 6

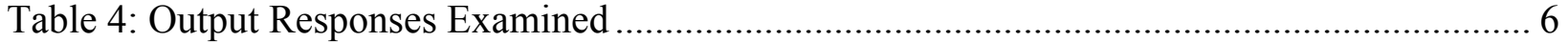




\section{Executive Summary}

In recent years, the wind industry has sponsored the development, verification, and validation of comprehensive aeroelastic simulators, which are used by industry, academia, and government entities for wind turbine design, certification, and research. Unfortunately, as wind turbines continue to grow in size, become more flexible, are augmented with sophisticated controllers, and sometimes exhibit unconventional design characteristics, the existing codes do not always support the additional analysis features required for proper design. Examples of analysis options not supported by most wind turbine design codes include aeroelastic stability, a wide variety of aerodynamic modeling options, well-integrated control implementation, modal reduction, and others. These limitations and the increasing need to perform more advanced analyses are motivating the wind industry to search for new and improved analysis tools.

The development history, functionality, and advanced nature of RCAS (Rotorcraft Comprehensive Analysis System) make this code a sensible option. RCAS is an aeroelastic simulator developed over a 4-year cooperative effort among the U.S. Army's Aeroflightdynamics Directorate, Advanced Rotorcraft Technology (ART), Inc., and the helicopter industry. An additional 14 years were spent developing its predecessor. As its name suggests, RCAS was created for the rotorcraft industry but developed as a general purpose code for modeling the aerodynamic and structural response of any system with rotating and nonrotating subsystems (such as wind turbines). RCAS employs the finite element method (FEM) modeling approach. It includes nonlinear beam "elements" capable of modeling important centrifugal, gyroscopic, large-deflection, and pretwist effects needed for accurate rotor blade modeling. Several rotary aerodynamic modules are available, including blade-element-momentum (BEM), lifting line, prescribed wake, free wake, and the capability of modeling aerodynamic interactions among the rotor, nacelle, and tower. RCAS is easily integrated with advanced controls in a familiar Simulink ${ }^{\circledR}$-style environment, and it incorporates many features not available in existing wind turbine analysis codes, including aeroelastic stability analysis, modal reduction, and periodic Floquet analysis.

To demonstrate that RCAS can analyze wind turbines, models of a conventional, 1.5-MW, 3-bladed, upwind, horizontal axis wind turbine (HAWT) are created in RCAS and wind-industry-accepted wind turbine analysis codes FAST (Fatigue, Aerodynamics, Structures, and Turbulence) and ADAMS (Automatic Dynamic Analysis of Mechanical Systems). Using these models, a side-by-side comparison of structural response predictions is performed under several test scenarios. The project scope is limited to a high-level comparison of the systems responses under a few, typical wind turbine analysis conditions; a detailed verification of low-level element responses is not developed. Furthermore, the study does not attempt to demonstrate the diverse functionality of RCAS; only the basic structural dynamic features are employed.

All three codes employ different modeling techniques. Nevertheless, comparisons of response predictions among the codes show excellent agreement and do not expose any glaring inaccuracies in RCAS. For example, modal analyses of the ADAMS and RCAS models show that the predicted full-system natural frequencies are within $2 \%$ of each other for at least the first 15 modes. Regions where the different response predictions do not exactly coalesce are attributed to differences in modeling techniques, such as integration methods and the differences between the FEM (RCAS), assumed modes (FAST), and lumped-properties (ADAMS) structural dynamics modeling approaches.

The wind industry's acceptance and acquisition of RCAS are not without obstacles, however. RCAS's inherent complexity is a mixed blessing, and its user interface is somewhat lacking. The learning curve is also steep. Nevertheless, the user-friendliness will naturally improve in time as the code and its user's manuals are upgraded. In the end, the wind industry must decide whether the gains accrued from RCAS's enhanced functionality relative to existing wind turbine analysis tools outweigh the costs of adopting this new code. 


\section{Background}

Over the past decade, the U.S. Department of Energy's (DOE's) National Renewable Energy Laboratory (NREL) has sponsored the development, verification, and validation of comprehensive aeroelastic simulators capable of predicting both the extreme loads and the fatigue life of wind turbines. These simulation tools, also known as design codes, are used by industry, academia, and government entities for wind turbine design, certification, and research. In general, these design codes enable a user to (1) define an aerodynamic and structural model of a wind turbine given the turbine geometry and aerodynamic and mechanical properties of its members, and (2) simulate the wind turbine's aerodynamic and structural response by imposing complex, virtual wind-inflow conditions. Outputs of the simulations include time-series data on the loads and deflections of the structural members of the wind turbine. Post-processing codes are then used to analyze these data.

In many respects, design codes bridge the gap between theorized predictions and experimental and/or observable measurements. Design codes enable virtual experiments capable of yielding load analysis results quickly and cheaply. In many situations, virtual experimentation offers the only practical method of research and testing.

FAST [1] and ADAMS ${ }^{\circledR}$ [2] are two of the most sophisticated codes used by the U.S. wind industry and the two most promoted by NREL's National Wind Technology Center (NWTC). ADAMS is a commercially available, general purpose, multibody-dynamics code from MSC.Software Corporation that is adaptable for modeling wind turbines. FAST is a structural-response, wind-turbine-specific code originally developed by Oregon State University and the University of Utah for the NWTC. Both FAST and ADAMS use Windward Engineering LLC's AeroDyn aerodynamic subroutine package for calculating aerodynamic forces [3].

Unfortunately, as wind turbines continue to grow in size, become more flexible, are augmented with sophisticated controllers, and sometimes exhibit unconventional design characteristics, the existing codes do not always support the additional features required for proper design. Examples of options not supported by most wind turbine design codes (including FAST and ADAMS) include aeroelastic stability, multiple aerodynamic modeling options, wellintegrated control implementation, modal reduction, and others.

The RCAS aeroelastic code [4], [5], [6], the successor of the Second Generation Comprehensive Helicopter Analysis System (2GCHAS), has the potential to fill this void. This code is a result of a 4-year cooperative effort among the U.S. Army's Aeroflightdynamics Directorate, Advanced Rotorcraft Technology (ART), Inc., and the helicopter industry. An additional 14 years were spent developing 2GCHAS. RCAS was created for the rotorcraft industry but developed as a general purpose code for modeling the aerodynamic and structural response of any system with rotating and nonrotating subsystems (such as wind turbines).

There are many motivations for exploring the potential to use RCAS for analyzing wind turbines. It is the most advanced aeroelastics code available for analyzing rotorcraft technology, with almost 20 years of development history. It includes nonlinear beam elements capable of modeling important centrifugal, gyroscopic, largedeflection, and pretwist effects needed for accurate rotor blade modeling. The code is flexible enough to model unconventional, precurved blades, rotors with teeter and delta-3, and complex linkages for blade collective pitch control. Several rotary aerodynamic modules are available, including blade-element-momentum (BEM), lifting line, prescribed wake, free wake, and the capability of modeling aerodynamic interactions among the rotor, nacelle, and tower. RCAS is easily integrated with advanced controls in a familiar Simulink ${ }^{\circledR}$-style environment, and it incorporates many features not available in existing wind turbine analysis codes, including aeroelastic stability analysis, modal reduction, and periodic Floquet analysis. Finally, RCAS is free to U.S. industries, unlike many commercial codes (such as ADAMS), which require expensive licensing fees.

\section{Objective}

The objective of this study is to demonstrate that RCAS can analyze wind turbines through a side-by-side comparison of response predictions obtained using RCAS and the industry-accepted wind turbine analysis codes FAST and ADAMS. The project scope is limited to a high-level comparison of the systems responses under a few, typical wind turbine analysis conditions; a detailed verification of low-level element responses is not developed 
herein. Furthermore, the study does not attempt to demonstrate the diverse functionality of RCAS; only the basic structural dynamic features are employed.

\section{Approach}

A conventional, 1.5-MW, 3-bladed, upwind, horizontal-axis wind turbine is selected for the side-by-side comparison. Models of this turbine are created using RCAS and the industry-accepted wind turbine analysis codes FAST and ADAMS. Operating cases are chosen to demonstrate that RCAS can be applied to analyze wind turbines, and the results are compared in a side-by-side fashion.

\section{Description of Codes}

FAST, ADAMS, and RCAS all employ different techniques of modeling wind turbine structural dynamics. This section documents the general class of modeling techniques employed in the various codes. For a more detailed description of the structural dynamic theories each code employs, please refer to their respective user's guides and theory manuals [1], [2], [4], [5], [6]. Modeling tools continue to be upgraded and versions of the codes become outdated; for reference, Table 1 lists the programs and version numbers used in this study.

Table 1: Program Versions Used in this Study

\begin{tabular}{|l|l|}
\hline Program & Version \\
\hline FAST & 4.10 (not yet released to public), AeroDyn 12.51 \\
\hline ADAMS & 12.0 .0$, AeroDyn 12.51 \\
\hline RCAS & $1.9 .5 \mathrm{a}$ \\
\hline
\end{tabular}

FAST is the simplest of the three codes used in this study and was developed specifically for modeling horizontalaxis wind turbines [1]. It has a limited number of degrees of freedom (DOFs) but can model most common wind turbine configurations and control scenarios. FAST models the blades and tower as individual flexible elements using a modal representation. The flexibility characteristics of these members are determined by specifying distributed stiffness and mass properties along the span of the members and by prescribing their mode shapes through equivalent polynomial coefficients. Flexibility in the drive train is modeled using an equivalent linear spring and damper model. The high-speed shaft (HSS) inertias and torques are cast to the low-speed shaft (LSS) through appropriate multiplications and divisions with the gearbox ratio, so the HSS is, in essence, not modeled independently of the LSS. The nacelle and hub are modeled in FAST as rigid bodies with appropriate mass and inertia terms. Time marching of the equations of motion (EoM) is performed using a constant-time-step, AdamsBashforth-Adams-Moulton, predictor-corrector integration scheme.

The structural dynamics models of ADAMS are more sophisticated, permitting virtually an unlimited combination of model configurations and DOFs [2]. It is not a wind-turbine-specific code and is routinely used by members of the automotive, aerospace, and robotics industries. Flexible members, such as the blades and tower of a wind turbine, are modeled in ADAMS using a series of lumped masses connected by flexible "fields" akin to multidimensional spring dampers. ADAMS can model the drive train through a similar series of lumped masses and flexible fields or through a simple, single-DOF hinge/spring/damper element. As in FAST, the nacelle and hub are usually modeled using rigid bodies with lumped mass and inertia properties but can be modeled with flexibility. ADAMS incorporates a similar time-marching integration scheme as the one in FAST, except that the ADAMS scheme incorporates a variable time step algorithm.

RCAS is the most complex of the three codes, employing the FEM approach [4], [5], [6]. Instead of an assumedmodes or lumped-mass approach, flexible members in RCAS are modeled as fully flexible beam elements with fully distributed mass and stiffness properties. As in typical FEM work, the model is assembled by defining "nodes," which are then interconnected by "elements." The types of "elements" range from the trivial single-DOF hinge/spring/damper element to the complex, fully flexible, nine-DOF nonlinear beam element". The nonlinear

\footnotetext{
${ }^{\dagger}$ To be precise, the nonlinear beam element actually has 15 DOF if it is not cantilevered to a parent element.
} 
beam element takes into account important centrifugal, gyroscopic, and large-deflection effects needed for accurate rotor blade modeling. The flexibility characteristics of the nonlinear beam elements are determined by prescribing distributed stiffness and mass properties along the span of the elements, similar to the input format used to define a FAST model; these are then integrated along the beam elements using Gaussian Quadrature integration techniques. Time marching of the EoM is achieved using a constant time step, Hilber-Hughes-Taylor (HHT) integration scheme, which is based on the Newmark-Beta integration method.

Both FAST and ADAMS use the AeroDyn aerodynamic subroutine package for computing aerodynamic forces [3]. This aerodynamic module package models wind turbine aerodynamics using the classic, equilibrium-based, BEM theory or by using a generalized dynamic inflow model, both of which include the effects of axial and tangential induction and tip and hub losses as characterized by Prandtl. Dynamic stall behavior is characterized using the Beddoes-Leishman dynamic stall model.

Previous comparisons among FAST (previously known as FAST_AD in some literature), ADAMS, and other industry-accepted wind turbine analysis codes show excellent agreement between FAST and ADAMS response predictions [7], [8], [9]. RCAS is just recently entering the validation phase. Some initial findings associated with the structural mechanics and dynamics of rotor blades show excellent promise [10]. RCAS's predecessor 2GCHAS has also been extensively validated.

\section{Turbine Description}

The turbine geometry, mechanical properties, and aerodynamic information were obtained from the baseline turbine in the Wind Partnership for Advanced Component Technologies (WindPACT) Turbine Rotor Design Study [11]. The WindPACT Rotor Design Study was performed by Global Energy Concepts and Windward Engineering Inc. to identify technology improvements that will enable the cost of energy from wind turbines to fall to a target of $3.0 \notin / \mathrm{kWh}$ in low-wind-speed sites.

The WindPACT baseline turbine was created by surveying modern wind turbines. It represents a conventional, 1.5MW, 3-bladed, upwind, horizontal-axis wind turbine. Table 2 contains a summary of the turbine design properties.

Table 2: Summary of Baseline Turbine Design Properties

\begin{tabular}{|l|l|}
\hline Rotor Diameter & $70 \mathrm{~m}$ \\
\hline Hub Height & $84 \mathrm{~m}$ \\
\hline Hub Overhang & $3.3 \mathrm{~m}$ \\
\hline Rotor Precone & $0^{\circ}$ \\
\hline Shaft Tilt & $5^{\circ}$ \\
\hline Tower Base Diameter & $5.663 \mathrm{~m}$ \\
\hline Max Rotor Speed & $20.5 \mathrm{rpm}$ \\
\hline $\begin{array}{l}\text { Tip Speed Ratio for Maximum } \\
\text { Power Coefficient }\end{array}$ & 7.0 \\
\hline
\end{tabular}

A working ADAMS model of the WindPACT baseline turbine was obtained from Windward Engineering Inc. This ADAMS model was used to verify the NREL FAST and ADAMS models used in this study through a side-by-side comparison of response predictions. In performing this model comparison, several differences and a few errors were discovered in Windward Engineering's original model. Although the results of this model comparison are not documented in this report, three small errors in Windward Engineering's model are reported here to assist others who might wish to use the Windward Engineering WindPACT baseline model. Two of the errors concern the blade and tower inertias. On the blade, the distributed flap and edge inertias are switched. On the tower, the distributed tower inertia is twice that indicated in the turbine description. The third error is that each blade mass is $1000 \mathrm{~kg}$ heavier in Windward Engineering's ADAMS model than stated in the turbine description. This is due to poor interpolation of the distributed blade mass near the blade root section. 


\section{Description of Models}

The FAST code can model a three-bladed HAWT with a maximum of 17 DOFs. All DOFs but the nacelle tilt and free yaw are enabled in the FAST model used in this study. These include blade flexibility (two flap and one edge mode for each independent blade), tower flexibility (two fore-aft and two side-to-side modes), and drive train torsion. The generator side of the shaft compliance is forced to spin at a constant rate (removing an additional available DOF), eliminating the need to implement electrical generator models, which are deemed unnecessary for the comparison of the various code's structural response predictions. A program entitled "Modes" [12] is used to generate the mode shapes for the assumed modes of the flexible blades and tower. An example set of FAST input files are given in Appendix A for the turbine spinning in a vacuum.

As alluded to in Table 1, a recently updated version of FAST, which has not yet been released to the public, is used in this study. This version of FAST has the capability of extracting "equivalent" ADAMS wind turbine datasets from the turbine properties specified in the FAST input file(s). That is, this new version of FAST has the functionality of acting like an ADAMS-preprocessor capable of creating ADAMS datasets of wind turbine models through FAST's simple property-input-style interface. The main advantages of using FAST to create the ADAMS model are to ensure consistency between the FAST and ADAMS models and to facilitate quick and easy creation of the ADAMS datasets.

The ADAMS datasets extracted from FAST contain most of the functionality and usability associated with the FAST model, while bypassing some of FAST's limitations. For example, instead of applying the assumed-mode characteristics of FAST's flexible members, the blades and tower of the extracted ADAMS model are developed from FAST's distributed mass and stiffness inputs using ADAMS' conventional approach of a series of lumped masses, connected by stiffness and damping fields. Additionally, several characteristics not implemented in the FAST model are incorporated into the extracted ADAMS model. Two notable examples are the distributed blade mass and elastic offsets. These offsets are incorporated into the ADAMS blade model, whereas the FAST model assumes the center of mass (c.m.) and elastic axes to be coincident with the collective pitch axis.

Like the FAST model, the yaw and tilt DOFs are disabled in the ADAMS model and the generator (cast to the LSS) is forced to rotate at a constant speed (though drive train torsion compliance is included). Tower flexibility is modeled in ADAMS using 10 equally spaced lumped masses (plus a yaw bearing mass) interconnected by 11 linear stiffness and damping fields. Similarly, each blade is modeled using 15 equally spaced lumped masses (plus a tip mass) interconnected by 16 linear stiffness and damping fields. The total number of structural DOFs in the ADAMS model is 355 .

The RCAS model is developed so as to replicate the ADAMS model as close as possible (which, in turn, replicates the FAST model as close as possible). To do this, mass, stiffness, and geometrical properties are prescribed identically among all three models. Also, the nodes of the RCAS model are located where the lumped masses are positioned in the ADAMS model. In the RCAS blade models, these nodes are interconnected by 16 nonlinear beam elements, similar to the 16 ADAMS stiffness and damping fields. In the RCAS tower model, the nodes are interconnected by 11 nonlinear beam elements, similar to the 11 ADAMS stiffness and damping fields. The three internal nodes of each nonlinear beam element used in the RCAS model are disabled. Drive train compliance is modeled in RCAS using a single-DOF hinge/spring/damper element, and the generator is again cast to the LSS. Control hinges are implemented at the three blade collective pitch bearings and the nacelle yaw bearing so that motions of these hinges could easily be prescribed during the analysis phase. Like ADAMS, the RCAS model has 355 DOFs.

Regardless of the analysis type, all models are created so that their simulations begin with no initial deflection of their flexible members. This should be evident in the response comparisons documented in the following section, but it is constructive to reiterate this point: The simulations do not begin at static or dynamic equilibrium. In FAST, this is intrinsic, as the model begins with all DOFs zero-valued at time zero (unless initial conditions are prescribed otherwise in FAST's primary input file). In ADAMS, this is achieved by "locking" all DOFs over the first integration time step. In RCAS, this is achieved by prescribing all DOFs and their derivatives to equal zero before the time-series analysis (known as a "maneuver" analysis in RCAS) is initiated. 


\section{Simulation Results}

The code demonstration consisted of five analyses: 1) parked turbine modal analysis, 2) spinning in a vacuum, 3) spinning and yawing in a vacuum, 4) steady loads, and 5) sinusoidal loads. Figure 1 depicts these analyses. In the last two analyses, point loads in two directions (normal and tangential) are applied to all 15 of the blade nodes in ADAMS and RCAS to simulate the effect of aerodynamic loads and associated responses. FAST results are not included for the last three analyses, due to the inherent difficulty in performing these types of analyses in FAST.
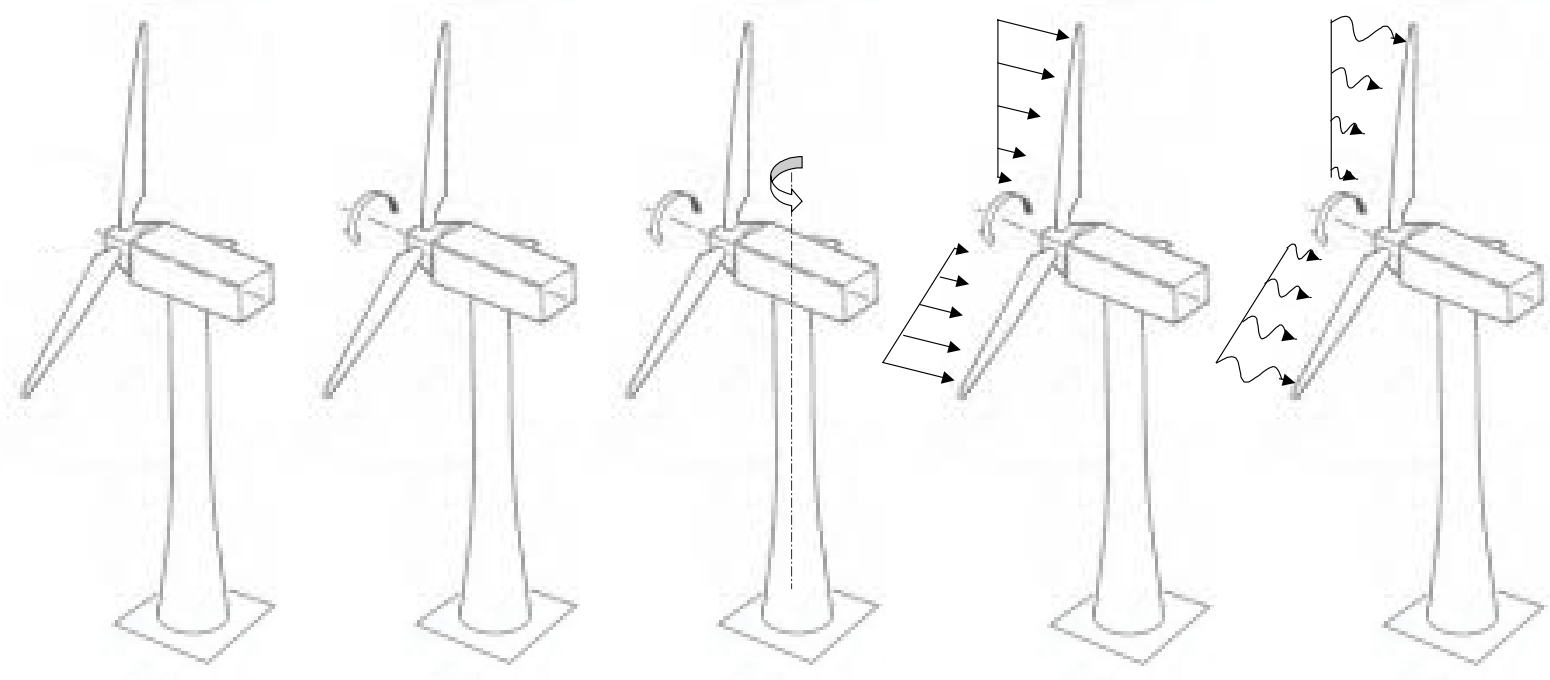

Figure 1: Graphical representation of analyses performed in the code demonstration.

\section{Parked Turbine Modal Analysis}

The modal analysis is performed on a stationary turbine (not spinning) and ignores gravitational and aerodynamic loads and structural damping. The blade collective pitch angle of all three blades is set at $2.6^{\circ}$, which corresponds with the minimum set position. The first 15 full system natural frequencies and mode shapes are examined. The resulting frequencies are listed in Table 3 along with a description of the corresponding modes. In ADAMS, these are obtained by invoking an ADAMS "LINEAR/EIGENSOL" command, which linearizes the complete ADAMS model and computes eigendata. In RCAS, a similar solution procedure is invoked using a stability analysis. For the FAST model, only independent system natural frequencies (i.e., tower alone or blade alone) are available outputs due to current limitations in the code. These frequencies are compared to the closest matching full system modes from ADAMS and RCAS in Table 3 for reference only. The types of the modes were established by viewing animations of the modes in ADAMS/View and verifying them against deflection shapes available in an RCAS postprocessor. 
Table 3: Non-Rotating Full System Natural Frequencies

\begin{tabular}{|c|c|c|c|l|}
\hline Mode & FAST & ADAMS & RCAS & Description \\
\hline 1 & 0.421 & 0.408 & 0.405 & 1st tower side-to-side \\
\hline 2 & 0.421 & 0.409 & 0.407 & 1st tower fore-aft \\
\hline 3 & - & 1.151 & 1.154 & 1st blade axisymmetric flap (2 and 3 out of phase, 1 stationary) \\
\hline 4 & 1.236 & 1.203 & 1.206 & 1st blade axisymmetric flap (2 and 3 in phase, 1 out of phase) \\
\hline 5 & - & 1.256 & 1.259 & 1st blade symmetric flap \\
\hline 6 & - & 1.598 & 1.618 & 1st blade symmetric edge \\
\hline 7 & 1.878 & 1.824 & 1.860 & 1st blade axisymmetric edge (2 and 3 out of phase, 1 stationary) \\
\hline 8 & - & 1.852 & 1.888 & 1st blade axisymmertic edge (2 and 3 in phase, 1 out of phase) \\
\hline 9 & - & 2.824 & 2.832 & 1st blade 1 flap / tower fore-aft coupling \\
\hline 10 & - & 2.987 & 2.999 & Complicated blade flap and edge / tower coupling \\
\hline 11 & - & 3.262 & 3.278 & 2nd blade axisymmetric flap (2 and 3 out of phase, 1 stationary) \\
\hline 12 & 3.722 & 3.642 & 3.680 & 2nd blade axisymmetric flap (2 and 3 in phase, 1 out of phase) \\
\hline 13 & - & 3.730 & 3.766 & 2nd blade symmetric flap \\
\hline 14 & - & 5.557 & 5.578 & 2nd blade symmetric edge \\
\hline 15 & - & 5.992 & 6.026 & Complicated blade flap and edge / tower coupling \\
\hline$\dagger$ Single system natural frequencies (i.e., tower alone or blade alone); compared with the closest matching full system mode \\
\hline
\end{tabular}

In general, the predictions of full system natural frequencies agree very well among the models. The RCAS frequencies are within $2 \%$ of the ADAMS frequencies. These slight discrepancies are most likely due to differences in the models caused by the methods of integration and interpolation to find the blade and tower stiffness properties (more evidence of this will be demonstrated later). The FAST frequencies are within $3.2 \%$ of the ADAMS frequencies. These larger discrepancies result from modeling components decoupled (not integrated with the complete system) and don't characterize the full system responses that will result from a time-series analysis.

\section{Spinning in a Vacuum}

This analysis considers the turbine spinning in a vacuum (no aerodynamic effects) loaded only by gravity. The turbine is simulated running at rated speed $(20.463 \mathrm{rpm}, 2.14288 \mathrm{rad} / \mathrm{sec})$ for a number of fixed blade collective pitch angles. From each simulation, 34 output responses are examined (see Table 4). Selected responses when all three blades have $2.6^{\circ}$ collective pitch angle are plotted in Figure 5. In general, very good agreement exists among the results from the three codes.

Table 4: Output Responses Examined

\begin{tabular}{|l|c|}
\hline \multicolumn{1}{|c|}{ Response } & Number of Components \\
\hline Hub-height wind speed and direction & 2 \\
\hline Blade 1 tip deflections & 2 \\
\hline Blade 1 collective pitch angle & 1 \\
\hline Blade 1 root loads & 6 \\
\hline Rotor azimuth angle and speed & 2 \\
\hline Shaft loads at hub (includes rotor torque and thrust) & 6 \\
\hline Yaw position & 1 \\
\hline Tower-top deflections at yaw bearing & 2 \\
\hline Tower-top loads at yaw bearing & 6 \\
\hline Tower-base loads & 6 \\
\hline
\end{tabular}

Figure 2 is a plot of the radial blade root forces for each code. Centrifugal forces of the rotating blade bring about the mean load, and the oscillating component is driven by gravity. Variations in the first couple of seconds of the simulation are attributed to the different simulation approaches and associated start-up transients ${ }^{\dagger}$. These are not of particular concern since most analysts avoid recording data associated with simulation start-up transients. Beyond the first 2 seconds, the FAST and ADAMS responses are nearly indistinguishable. The RCAS response has a slightly larger amplitude and mean than FAST and ADAMS. This response suggests that RCAS has internally computed a larger blade mass than FAST and ADAMS. This is understandable because RCAS's Gaussian Quadrature integration approach is more accurate at capturing the large mass density gradient at the inboard portion

$\dagger$ The large fluctuation in RCAS's response is most likely due to the HHT time-marching algorithm, which acts to remove high-frequency transients at the simulation start-up. 
of the blade than FAST's and ADAMS' linear interpolation/integration techniques. More evidence of this blade mass discrepancy can be found by examining the yaw bearing tilt moments and tower-top fore/aft deflections in Figure 5. The magnitudes of the tilt moment and fore-aft tower deflections in the RCAS model are larger than in the other two codes, indicating a heavier rotor. This is also consistent with the slightly larger oscillatory period of the tower fore-aft deflections in Figure 5 and the slightly lower tower fore-aft frequency seen in Table 3.

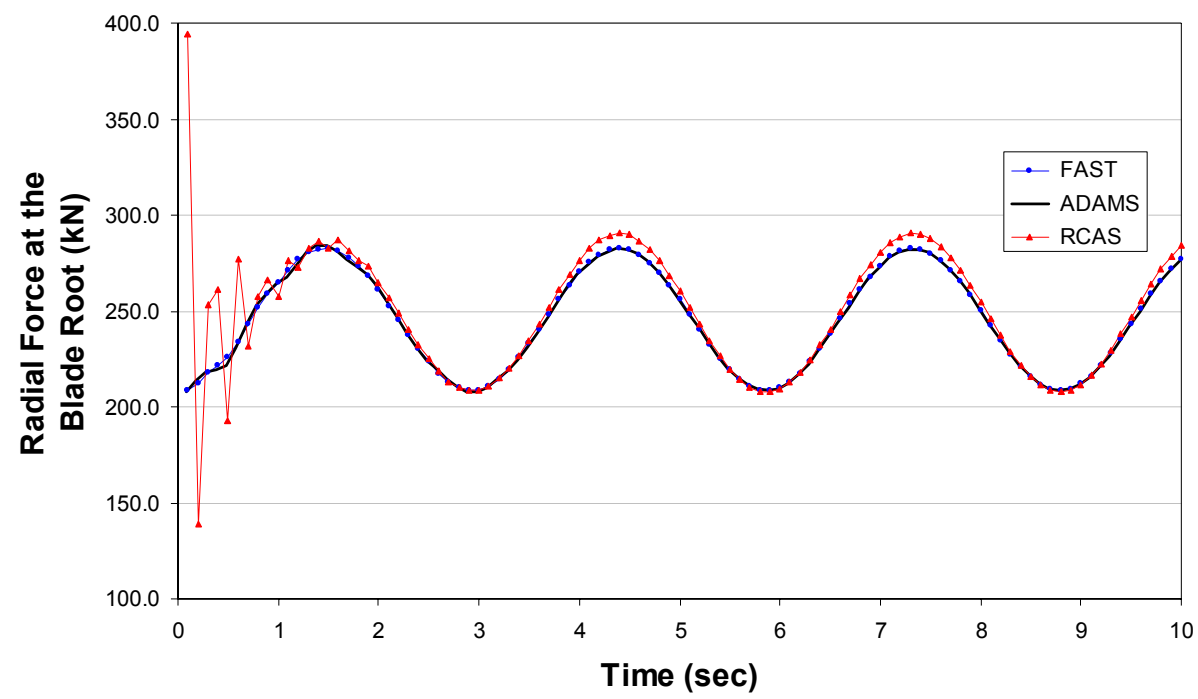

Figure 2: Radial blade root forces for the turbine spinning in a vacuum.

As discussed earlier in the description of models section of this report, the ADAMS and RCAS models include distributed elastic and center of gravity offsets in the blades, whereas these features are not available in FAST. The out-of-plane tip deflections in Figure 5 illustrate the effect that this limitation has on the FAST responses. The lower fidelity FAST model does not capture the resonances caused by these offsets.

\section{Spinning and Yawing in a Vacuum}

This analysis is identical to the previous one (spinning in a vacuum) except the nacelle and rotor are yawed at a rate of $0.75 \mathrm{deg} / \mathrm{sec}$ in the clockwise direction (looking downward) after an initial 10-second lapse in which the yaw angle is held fixed. The yaw angle as a function of time is plotted for each code in Figure 3. This case is run so that gyroscopically induced loads and deflections can be compared among the models. 


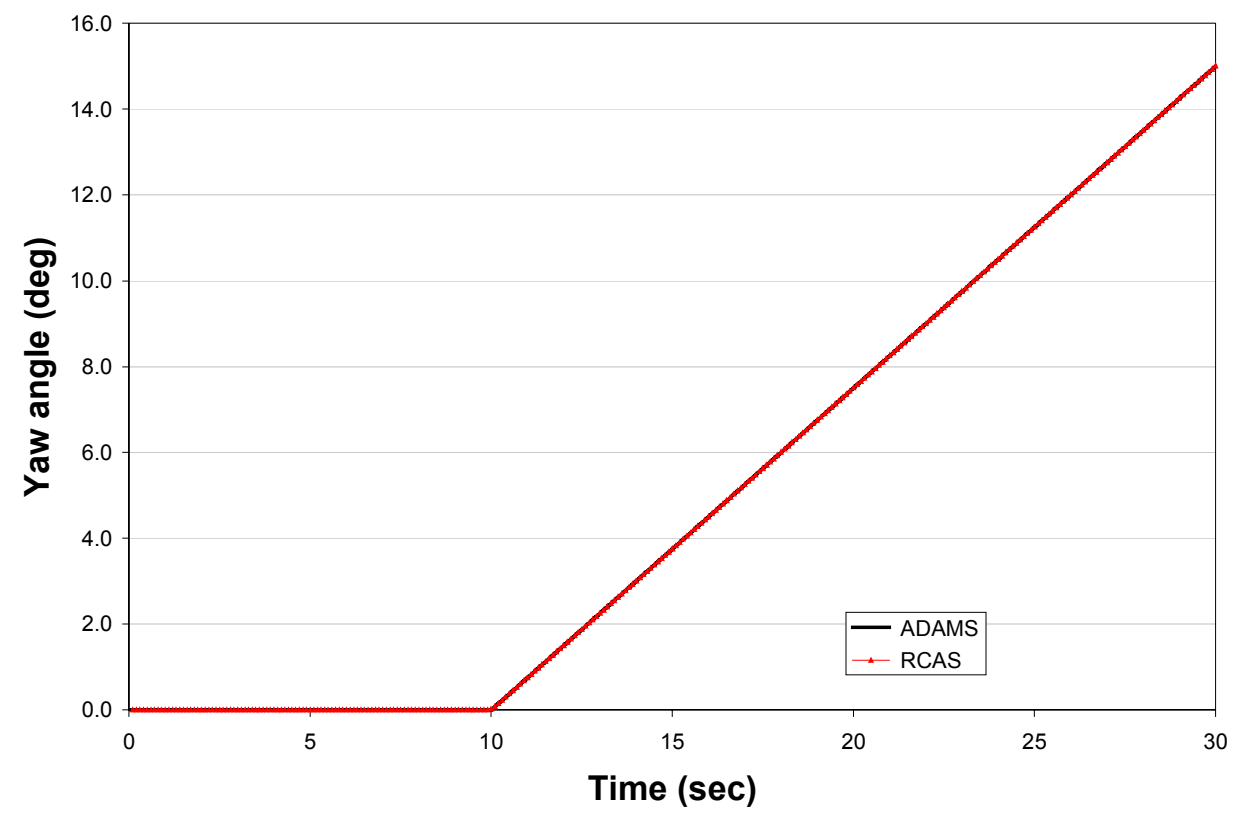

Figure 3: Output yaw angle for the turbine spinning and yawing in a vacuum analysis.

Selected responses are plotted in Figure 6. In general, there is good agreement between the RCAS and ADAMS response predictions, both before and after the nacelle yaw motion is initiated. Of particular interest in this case is the response of each code at the initiation of the yaw motion. The yaw motion is prescribed in ADAMS using a "MOTION" statement, which specifies the yaw rotation angular displacement as a function of time. When specifying a MOTION statement that prescribes a displacement in ADAMS, the code realizes that there must be an associated velocity and acceleration, and it applies appropriate time-derivates in the model accordingly. This approach results in an acceleration impulse at the instant the yaw motion begins (time $=10$ seconds) because the prescribed yaw position is not "smoothed out" at this point. This acceleration impulse "kicks" the system, which is evident in the tower-top yaw moment response of 10 seconds (Figure 4).

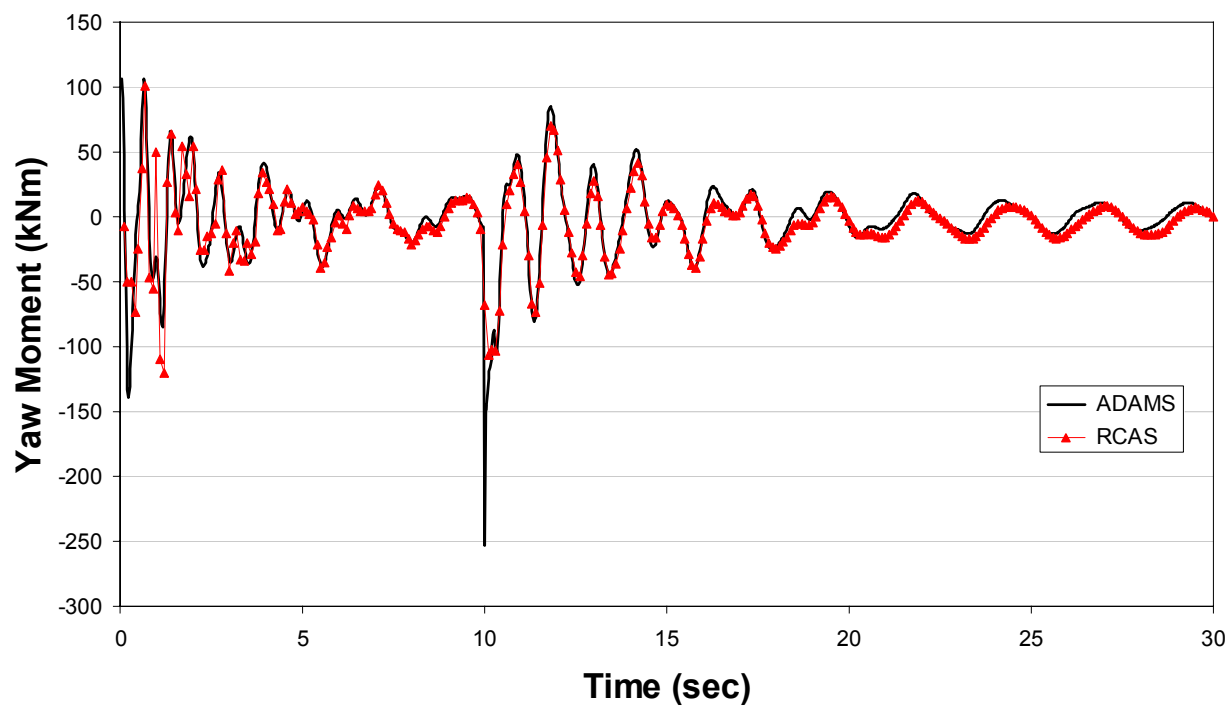

Figure 4: Yaw moment for the turbine spinning and yawing in a vacuum.

This "kick" is not evident in the RCAS response, which is explained as follows. Since the yaw bearing is modeled as a control hinge in RCAS (as discussed earlier in the description of models section of this report), the yaw motion 
in RCAS is prescribed through a time-varying control input in SCREEN "MANEUVERINPUT". The RCAS theory manual [4] clearly states that time-derivates of control inputs are not internally computed by RCAS, meaning in this case, that the yaw motion has no associated velocity, acceleration, or gyroscopically induced loads and deflections. Therefore, the responses would not correlate well between ADAMS and RCAS. To bypass these limitations, the yaw angle control input is passed through a second order actuator "element," which invokes RCAS to solve a differential equation for the yaw position, velocity, and acceleration that is forced by the desired control input yaw position. This results in a yaw response with appropriate velocity and acceleration that is a lagged and "smoothed" version of the desired control input. The "smoothed" behavior is evident in the lack of "kick" seen in Figure 4. The response lag is avoided for the side-by-side comparisons given in Figure 3, Figure 4, and Figure 6 by initiating the controlled yaw event earlier in RCAS than in ADAMS. An alternative method for modeling this situation in RCAS would be to specify not only the time-varying position of the yaw motion, but also the velocity and acceleration. The implementation of this alternative method is not included in this report.

\section{Steady Loads Analysis}

In this analysis, steady point loads are applied to all blade nodes in ADAMS and RCAS to simulate the effect of aerodynamic loads and associated responses. The point loads are applied to the ADAMS model via "GFORCE" statements. In RCAS, point loads are applied by adding mechanical load "elements" to the model (via SCREEN MECHLOAD). To obtain values for these loads, the FAST model (with AeroDyn) is run using a uniform, $12 \mathrm{~m} / \mathrm{s}$, steady wind input with no shear, turbulence, or yaw misalignment. The resulting normal and tangential forces in the AeroDyn output are averaged to determine the steady loads to apply at each node in the ADAMS and RCAS models.

Selected results are presented in Figure 7. As in previous cases, a very good agreement exists between the ADAMS and RCAS results. Slight variations in the results are attributed to the discrepancies in the blade mass and full system modal frequencies, as discussed earlier.

\section{Sinusoidal Loads Analysis}

This analysis expands on the steady load analysis by including the oscillatory components of the normal and tangential aerodynamic forces. The normal and tangential aerodynamic forces are oscillatory since the shaft tilt causes a periodic variation in the blade angle of attack as the blades advance and retreat relative to the wind. To obtain values for the point loads, amplitudes and means of the normal and tangential forces are computed at each node from the AeroDyn output of the FAST simulation with $12 \mathrm{~m} / \mathrm{s}$ winds. The phase angle of the normal component of the load at each node is also computed. For simplicity, the tangential components of the forces are assumed to oscillate in phase with the normal components. The amplitude, mean, and phase of each normal and tangential force at each node are then used to construct equivalent, harmonic point loads.

Selected results are presented in Figure 8. Again, the agreement between ADAMS and RCAS is very good. Moreover, there is little difference between these responses and those obtained from the steady point load analysis (Figure 7) because the oscillating component of the load is generally small relative to the mean component of the load. For example, the amplitude of the normal component is typically only $1 \%$ to $5 \%$ of the mean.

\section{A Critique of RCAS's Usability and Applicability in the Wind Industry}

The results illustrated above demonstrate that RCAS has the ability to model wind turbine structures as accurately as other industry-accepted codes do. Certainly, the response comparisons do not highlight any glaring weaknesses or inadequacies. However, the ability to obtain the "correct" results is not the only factor considered when adopting a new analysis tool. Factors such as code complexity, user friendliness, and code flexibility and functionality are equally important and will determine whether RCAS is accepted for use by the wind industry.

In terms of code complexity, RCAS is equally as complex as ADAMS. This is a direct result of RCAS's flexibility and implementation of the FEM modeling approach. In addition, RCAS is currently only in an alpha stage of development; little attention has been given to the graphical user interface. RCAS's input prompt-style interface and poor 3D-graphics capabilities also contribute to the code complexity, though the input prompt-style interface is somewhat bypassed through the use of script files and the 3D graphics capabilities will most likely improve with 
code upgrades in time. Nevertheless, first-time users of RCAS should expect a steep learning curve, just as they would for ADAMS. Background experience in structural dynamics analysis and the FEM modeling approach is a necessity. Programming experience with Fortran, C, and MATLAB are also important because the code is developed in these programming languages and because it is necessary to develop scripts in these languages (especially MATLAB) if one is to exploit the diverse functionality of RCAS ${ }^{\dagger}$.

With regard to user-friendliness and everyday usability, RCAS has both advantages and disadvantages when compared to ADAMS. RCAS's comparative advantages include a method for directly inputting distributed mass and inertia properties for determination of blade and tower flexibility, easy methods for implementing control paradigms, and its open source code, allowing for custom-tailoring by its users to suit their needs. One disadvantage is that the code was developed originally for the rotorcraft industry, and as such, the code utilizes sign conventions and terminology that are not always consistent with wind turbine lingo. This might bring about modeling mishaps if users trying to model wind turbines are not careful. Other drawbacks include the complicated interface, as discussed in the previous paragraph, and the fact that RCAS must be run on a Linux platform instead of on Windows. In general, the usability of RCAS in the wind industry may be improved upon by developing a wind-turbine-specific front end for the code.

One important weakness of the RCAS code, as noticed during this study, is the processing speed for a time-series response analysis. Time-series analyses using RCAS take roughly one order of magnitude longer than similar analyses in ADAMS for models with an identical number of DOF and time step size. This is most likely due to the sophisticated finite element methodology used in RCAS, as contrasted to the lumped-properties approach used in ADAMS. This may not be a serious limitation because FEM is considerably more accurate than the lumped method, and fewer DOFs in an RCAS model may suffice to yield a desired accuracy - thereby drastically reducing the process speed of an RCAS analysis. For example, users may be able to deactivate various states of individual nonlinear beam elements or use fewer elements if they are not important for the problem at hand.

Code functionality is another factor affecting the code's acceptance by the wind industry. RCAS's diverse capabilities, many of which are listed in the background section of this report, are one motivation for this study. One feature not yet available is bending-torsion coupling in the blades. This limitation may be important, as passive load control is currently an active research topic in the wind industry. However, with RCAS's open source format and custom-tailoring potential, this limitation may be remedied with some work. In fact, ART Inc. currently plans to introduce anisotropic composite beam elements into the code. Integration of RCAS with a wind-industryaccepted aerodynamics modeling package, such as AeroDyn, is another limitation that can be eliminated with some work.

\section{Conclusions}

Limitations in the existing design codes and the increasing need to perform more advanced analyses motivate the wind industry to search for new and improved analysis tools. The development history, functionality, and advancednature of RCAS make this code a sensible option. To demonstrate that RCAS can be applied to analyze wind turbine structures, a side-by-side comparison is performed of response predictions obtained using RCAS and industry-accepted wind turbine analysis codes FAST and ADAMS. All three codes employ different modeling techniques. Nevertheless, comparisons of response predictions between the codes show excellent agreement and do not expose any glaring inaccuracies in RCAS. Regions where the different response predictions do not exactly coalesce are attributed to differences in the models techniques, such as integration methods and the differences between the FEM, assumed-modes, and lumped-properties modeling approaches.

The wind industry's acceptance and acquisition of RCAS is not without obstacles, however. RCAS's inherent complexity is a mixed blessing, and its user interface is somewhat lacking. The learning curve is also steep.

\footnotetext{
$\dagger$ To be precise, much of RCAS is written in script files that closely resemble "m-files" used in MATLAB, and a large portion of RCAS's user environment behaves similarly to the MATLAB-style environment. However, in RCAS, this environment is called RSCOPE, which is a custom-designed environment and programming language developed by ART Inc. There is no association between RCAS and MATLAB.
} 
Nevertheless, the user-friendliness will naturally improve in time as the code and its user's manuals are upgraded. In the end, the wind industry must decide whether the gains accrued from RCAS's enhanced functionality relative to existing wind turbine analysis tools outweigh the costs of adopting this new code.

\section{Acknowledgements}

The authors would like to thank Hossein Saberi from ART, Inc. for the technical support he provided on the inner workings of RCAS. Additional thanks go to Marshall Buhl of NREL for his computer support for this project. Finally, the authors would like to thank Mike Robinson and Gunjit Bir of NREL for their guidance and funding for this study.

\section{References}

[1] Buhl Jr., M. L.; Jonkman, J. M.; Wright, A. D.; Wilson, R. E.; Walker, S. N.; Heh, P. FAST User's Guide, NREL/EL-500-29798. Golden, Colorado: National Renewable Energy Laboratory, 2002.

[2] Hansen, A. C.; Laino, D. J. User's Guide to the Wind Turbine Dynamics Computer Programs YawDyn and AeroDyn for ADAMS, University of Utah, 1998.

[3] Laino, D. J.; Hansen, A. C. User's Guide to the Wind Turbine Aerodynamics Computer Software AeroDyn, Windward Engineering, 2002.

[4] Anon. RCAS Theory Manual, Version 2.0, United States (US) Army Aviation and Missile COMmand/AeroFlightDynamics Directorate (USAAMCOM/AFDD) Technical Report (TR) 02-A-005, USAAMCOM/AFDD TR 02-A-005, U.S. Army Aviation and Missile Command, Moffett Field, CA, June 2002.

[5] Anon. RCAS User's Manual, Version 2.0, United States (US) Army Aviation and Missile COMmand/AeroFlightDynamics Directorate (USAAMCOM/AFDD) Technical Report (TR) 02-A-006, USAAMCOM/AFDD TR 02-A-006, U.S. Army Aviation and Missile Command, Moffett Field, CA, June 2002.

[6] Anon. RCAS Applications Manual, Version 2.0, United States (US) Army Aviation and Missile COMmand/AeroFlightDynamics Directorate (USAAMCOM/AFDD) Technical Report (TR) TR 02-A-007, U.S. Army Aviation and Missile Command, Moffett Field, CA, June 2002.

[7] Buhl Jr., M. L.; Wright, A. D.; Tangler, J. L. "Wind Turbine Design Codes: A Preliminary Comparison of the Aerodynamics." Prepared for the $17^{\text {th }}$ American Society of Mechanical Engineers (ASME) Wind Energy Symposium, January 12 - 15, 1998, Reno, Nevada. NREL/CP-500-23975. Golden, CO: National Renewable Energy Laboratory, December 1997.

[8] Buhl Jr., M. L.; Wright, A. D.; Pierce, K. G. "Wind Turbine Design Codes: A Comparison of the Structural Response." Proceedings, 2000 American Society of Mechanical Engineers (ASME) Wind Energy Symposium/38 $8^{\text {th }}$ American Institute of Aeronautics and Astronautics (AIAA) Aerospace Sciences Meeting and Exhibit, Reno, Nevada. AIAA-2000-0022, January 2000, pp. 12 - 22.

[9] Buhl Jr., M. L.; Wright, A. D.; Pierce, K. G. "FAST_AD Code Verification: A Comparison to ADAMS." Proceedings, 2001 American Society of Mechanical Engineers (ASME) Wind Energy Symposium $/ 39^{\text {th }}$ American Institute of Aeronautics and Astronautics (AIAA) Aerospace Sciences Meeting and Exhibit, Reno, Nevada. AIAA2000-0062, January 2001, pp. 368 - 377.

[10] Hopkins, A. S.; Ormiston, R. A. "An Examination of Selected Problems in Rotor Blade Structural Mechanics and Dynamics." Presented at the American Helicopter Society 59 th Annual Forum, May 6 - 8, 2003, Phoenix, Arizona. 
[11] Malcolm, D. J. (Global Energy Concepts, LLC); Hansen, A. C. (Windward Engineering). WindPACT Turbine Rotor Design Study: June 2000 - June 2002. NREL/SR-500-32495. Golden, CO: National Renewable Energy Laboratory, 2002.

[12] Buhl Jr., M. L. "A Simple Mode-Shape Generator for Both Towers and Rotating Blades," online, http://wind.nrel.gov/designcodes/modes/. Accessed February 2003. 

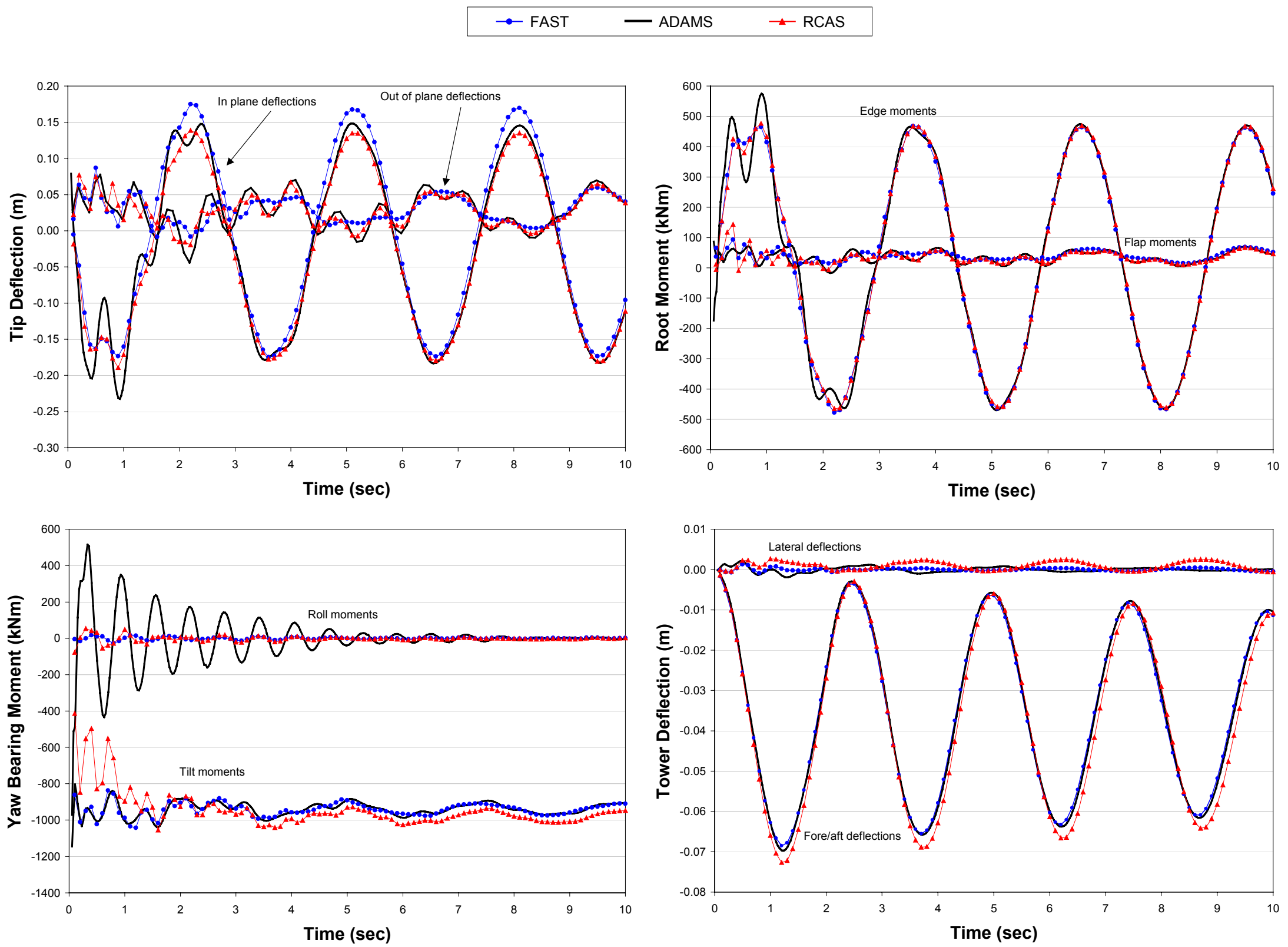

Figure 5: Selected responses for the turbine spinning in a vacuum.

13 

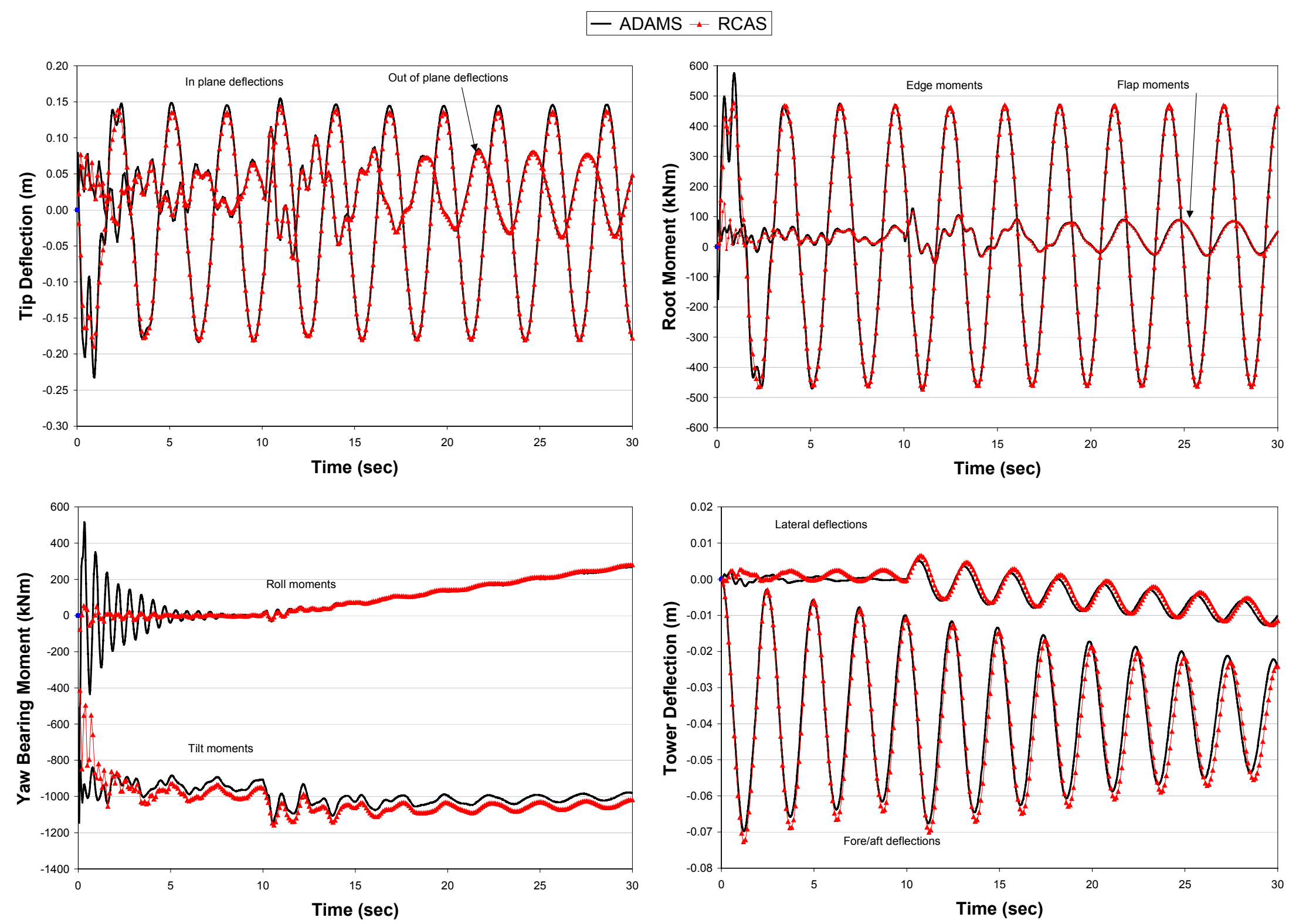

Figure 6: Selected responses for the turbine spinning and yawing in a vacuum. 

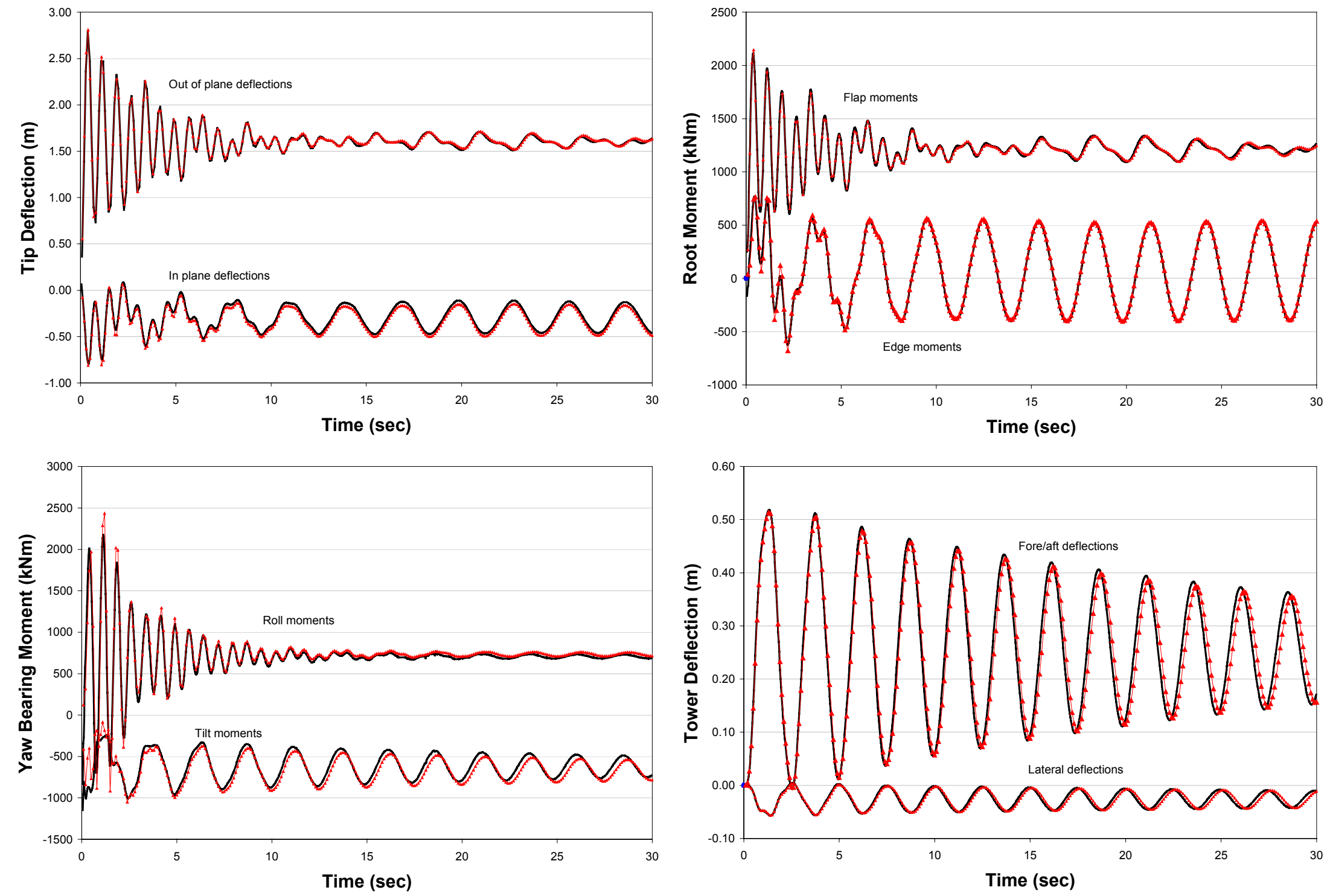

Figure 7: Selected responses for $12 \mathrm{~m} / \mathrm{s}$ steady loads applied to the turbine. 

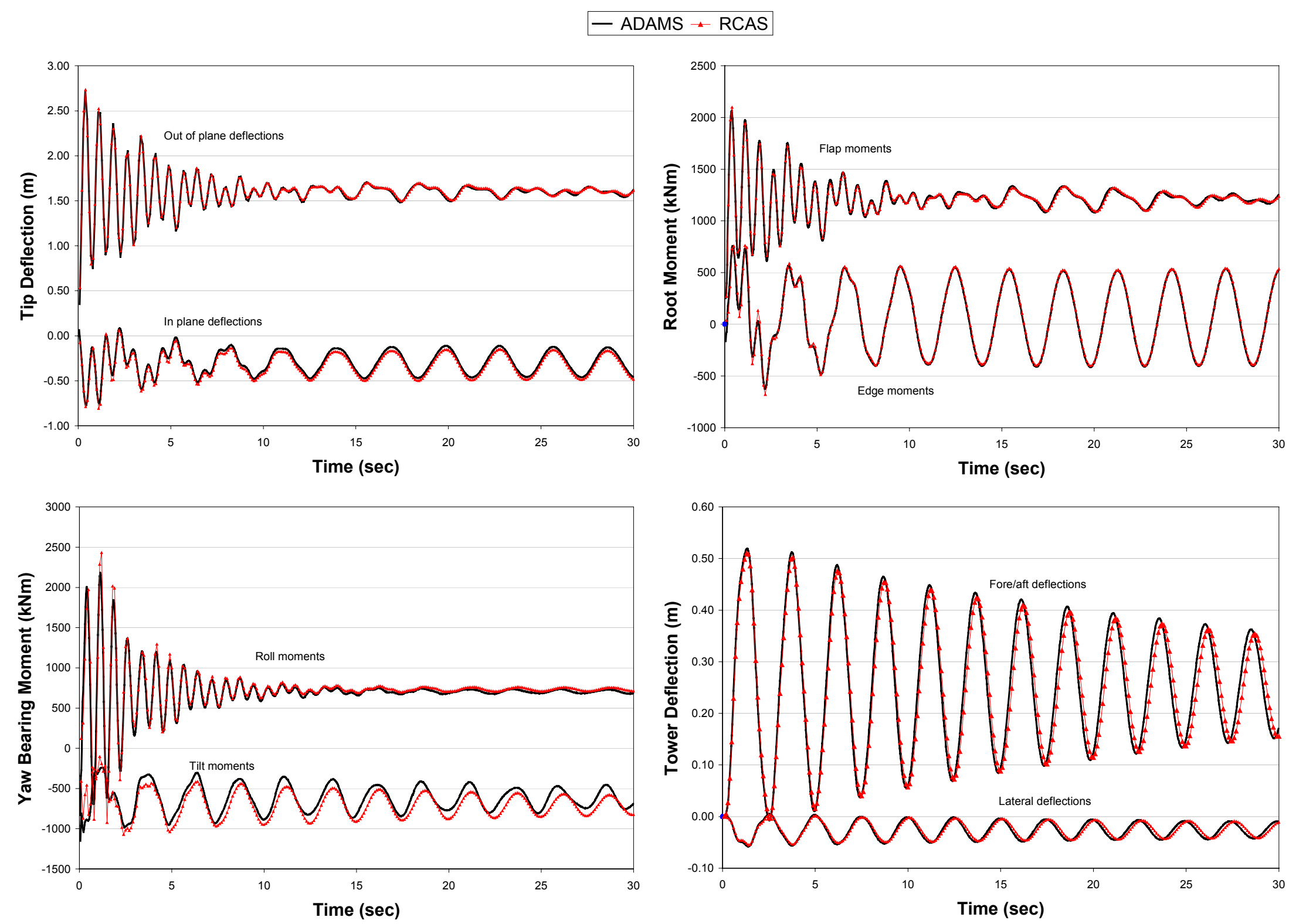

Figure 8: Selected responses for $12 \mathrm{~m} / \mathrm{s}$ sinusoidal loads applied to the turbine. 


\section{Appendix: Sample Input Files}

This appendix includes sample FAST and RCAS input files for the turbine spinning in a vacuum. The ADAMS Solver dataset is not included due to its length; it is available from the authors upon request.

\section{FAST Input File for the Turbine Spinning in a Vacuum}

-

FAST model of a 1.5 MW, 3-bladed, upwind, baseline turbine used for RCAS validation.

Model properties from "InputData1.5A08V07adm.xls" (from C. Hansen) with bugs removed. Compatible with FAST v4.1.

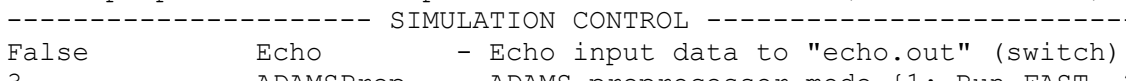

3 ADAMSPrep - ADAMS preprocessor mode \{1: Run FAST, 2: use FAST as a preprocessor to create an ADAMS model, 3: do both

(switch) NumBl - Number of blades $(-)$

10.0 NumBI - Number or blades $(-)$

0.005 DT $\quad$ - Integration time step (s)

$0 \quad$ PCMode $\quad$ - Pitch control mode $\{0$ : none, 1: power control, 2: speed control\} (switch)

9999.9 TPCOn - Time to enable active pitch control (s)

0 VSContrl - Variable-speed control \{0: none, 1: simple VS, 2: user-defined VS\} (switch)

9999.9 RatGenSp - Rated generator speed for simple variable-speed generator control (HSS side) (rpm) [used only when

9999.9 Reg2TCon - Torque constant for simple variable-speed generator control in Region 2 (HSS side) (N-m/rpm^2) [used only when VSContrl=1]

True

True

9999.9

0.0

9999.9

9999.9

9999.9

9999.9

9999.9

9999.9

9999.9

9999.9

9999.9

9999.9

99.

9999.9

blades

9999.9

9999.9

9999.9

2.6

$1]$

GenTistr

GenTistp

SpdGenon

TimGenon

Timgenof

THSSBrDp

TiDynBrk

TTpBrDp (1)

TTpBrDp (2)

TTPBrDp(3) - Time to initiate deployment of tip brake 3 (s) [unused for 2 blades]

TBDepISp(1) - Deployment-initiation speed for the tip brake on blade 1 (rpm)

TBDepISp(3) - Deployment-initiation speed for the tip brake on blade 3 (rpm) [unused for 2 blades]

TPitManS(1) - Time to start override pitch maneuver for blade 1 and end standard pitch control (s)

TPitManS(2) - Time to start override pitch maneuver for blade 2 and end standard pitch control (s)

TPitMans(3) - Time to start override pitch maneuver for blade 3 and end standard pitch control (s) [unused for 2

TPitManE(1) - Time at which override pitch maneuver for blade 1 reaches final pitch (s)

TPitManE(2) - Time at which override pitch maneuver for blade 2 reaches final pitch (s)

TPitManE(3) - Time at which override pitch maneuver for blade 3 reaches final pitch (s) [unused for 2 blades]

BlPitch(1) - Blade 1 initial pitch (degrees)

BlPitch(2) - Blade 2 initial pitch (degrees)

BlPitch(3) - Blade 3 initial pitch (degrees) [unused for 2 blades] 


\begin{tabular}{|c|c|c|}
\hline 2.6 & B1PitchF (1) & - Blade 1 final pitch for pitch maneuvers (degrees) \\
\hline 2.6 & B1PitchF (2) & - Blade 2 final pitch for pitch maneuvers (degrees) \\
\hline 2.6 & B1PitchF (3) & - Blade 3 final pitch for pitch maneuvers (degrees) [unused for 2 blades] \\
\hline 9.80665 & $\begin{array}{l}----- \text { ENVI } \\
\text { Gravity }\end{array}$ & $\begin{array}{l}\text { IRONMENTAL CONDITIONS }- \text { - } \\
\text { - Gravitational acceleration (m/s^2) }\end{array}$ \\
\hline & GIAVICY EEAT & TURE SWITCHES -- - \\
\hline True & FlapDOF1 & - First flapwise blade mode DOF (switch) \\
\hline True & FlapDOF2 & - Second flapwise blade mode DOF (switch) \\
\hline True & EdgeDOF & - First edgewise blade mode DOF (switch) \\
\hline False & TeetDOF & - Rotor-teeter DOF (switch) [unused for 3 blades] \\
\hline True & DrTrDOF & - Drivetrain rotational-flexibility DOF (switch) \\
\hline False & GenDOF & - Generator DOF (switch) \\
\hline False & TiltDOF & - Nacelle-tilt DOF (switch) \\
\hline False & YawDOF & - Yaw DOF (switch) \\
\hline True & TWFADOF1 & - First fore-aft tower bending-mode DOF (switch) \\
\hline True & TWFADOF2 & - Second fore-aft tower bending-mode DOF (switch) \\
\hline True & TTWSSDOF1 & - First side-to-side tower bending-mode DOF (switch) \\
\hline True & TwSSDOF2 & - Second side-to-side tower bending-mode DOF (switch) \\
\hline False & CompAero & - Compute aerodynamic forces (switch) \\
\hline & ----- INIT & TIAL CONDITIONS - - \\
\hline 0.0 & OoPDefl & - Initial out-of-plane blade-tip displacement, (meters) \\
\hline 0.0 & IPDefl & - Initial in-plane blade-tip deflection, (meters) \\
\hline 0.0 & TeetDefl & - Initial or fixed teeter angle (degrees) [unused for 3 blades] \\
\hline 0.0 & Azimuth & - Initial azimuth angle for blade 1 (degrees) \\
\hline 20.463 & RotSpeed & - Initial or fixed rotor speed (rpm) \\
\hline-5.0 & NacTilt & - Initial or fixed nacelle-tilt angle (degrees) \\
\hline 0.0 & NacYaw & - Initial or fixed nacelle-yaw angle (degrees) \\
\hline 0.0 & TTDspFA & - Initial fore-aft tower-top displacement (meters) \\
\hline 0.0 & TTDspSS & - Initial side-to-side tower-top displacement (meters) \\
\hline--- & $-----\mathrm{TU}$ & BINE CONFIGURATION - \\
\hline 35.0 & TipRad & - The distance from the rotor apex to the blade tip (meters) \\
\hline 1.75 & HubRad & - The distance from the rotor apex to the blade root (meters) \\
\hline & PSPnEIN & - Number of the innermost blade element which is still part of the pitchable portion of the blade for \\
\hline partial- & pitch control & {$[1$ to BldNodes] [CURRENTLY IGNORED] $(-)$} \\
\hline 0.0 & UndSling & - Undersling length [distance from teeter pin to the rotor apex] (meters) [unused for 3 blades] \\
\hline 0.0 & HubCM & - Distance from rotor apex to hub mass [positive downwind] (meters) \\
\hline-3.3 & OverHang & - Distance from yaw axis to rotor apex [3 blades] or teeter pin [2 blades] (meters) \\
\hline-0.1251 & ParaDNM & - Distance parallel to shaft from yaw axis to nacelle CM (meters) \\
\hline-0.2328 & PerpDNM & - Perpendicular distance from shaft to nacelle CM (meters) \\
\hline 82.39 & Towertt & - Height of tower above ground level (meters) \\
\hline 1.61 & Twr2Shft & - Vertical distance from the tower top to the yaw/shaft intersection (meters) \\
\hline 0.0 & TwrRBHt & - Tower rigid base height (meters) \\
\hline 0.0 & Delta3 & - Delta-3 angle for teetering rotors (degrees) [unused for 3 blades] \\
\hline 0.0 & Precone (1) & - Blade 1 cone angle (degrees) \\
\hline 0.0 & PreCone (2) & - Blade 2 cone angle (degrees) \\
\hline 0.0 & PreCone (3) & - Blade 3 cone angle (degrees) [unused for 2 blades] \\
\hline 0.0 & AzimB1Up & - Azimuth value to use for I/O when blade 1 points up (degrees) \\
\hline & & $=N 201]$ \\
\hline 51170.0 & NacMass & - Nacelle mass $(\mathrm{kg})$ \\
\hline 15148.0 & HubMass & - Hub mass (kg) \\
\hline & ipMass (1) & - Tip-brake mass, \\
\hline
\end{tabular}




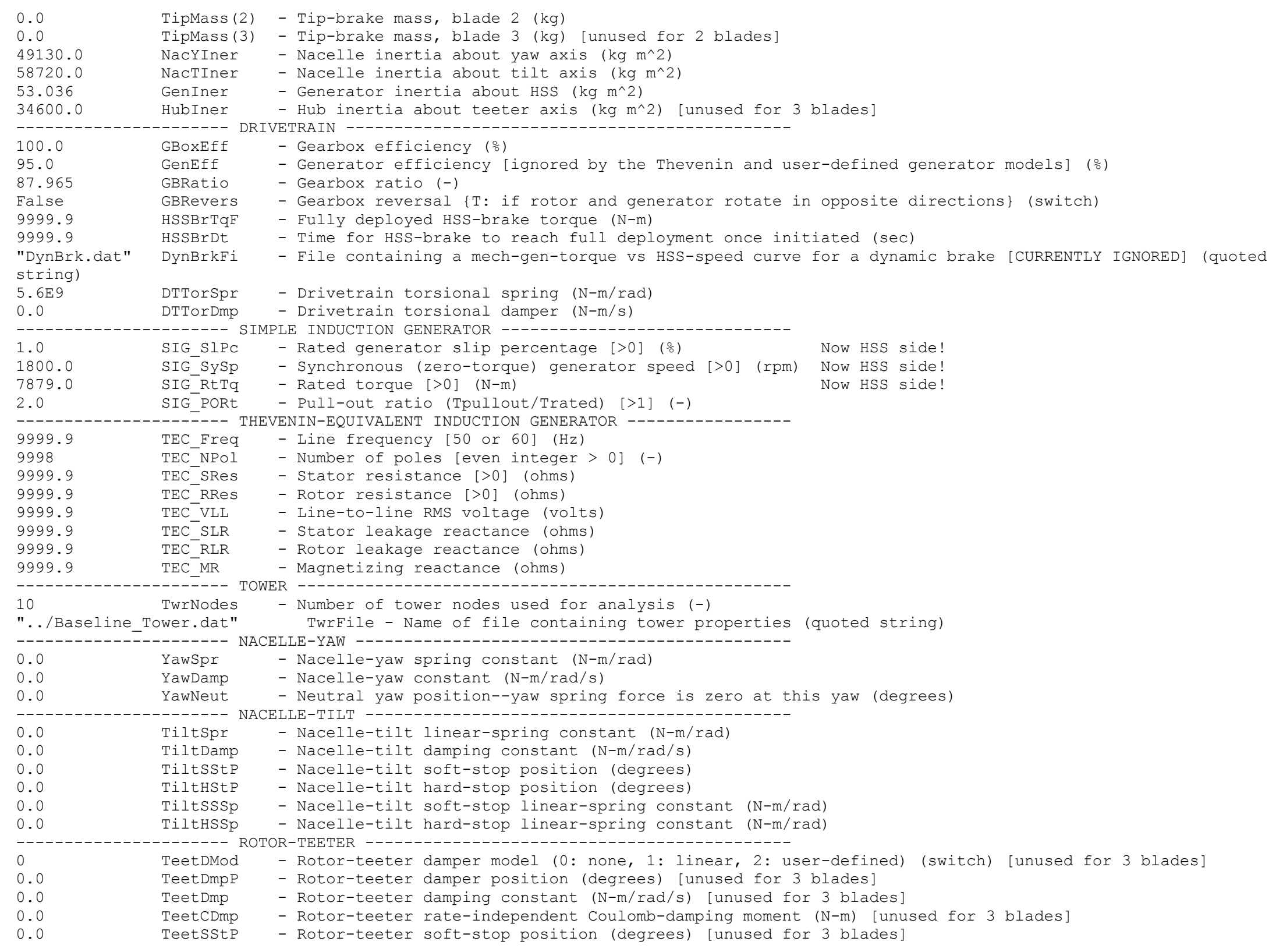




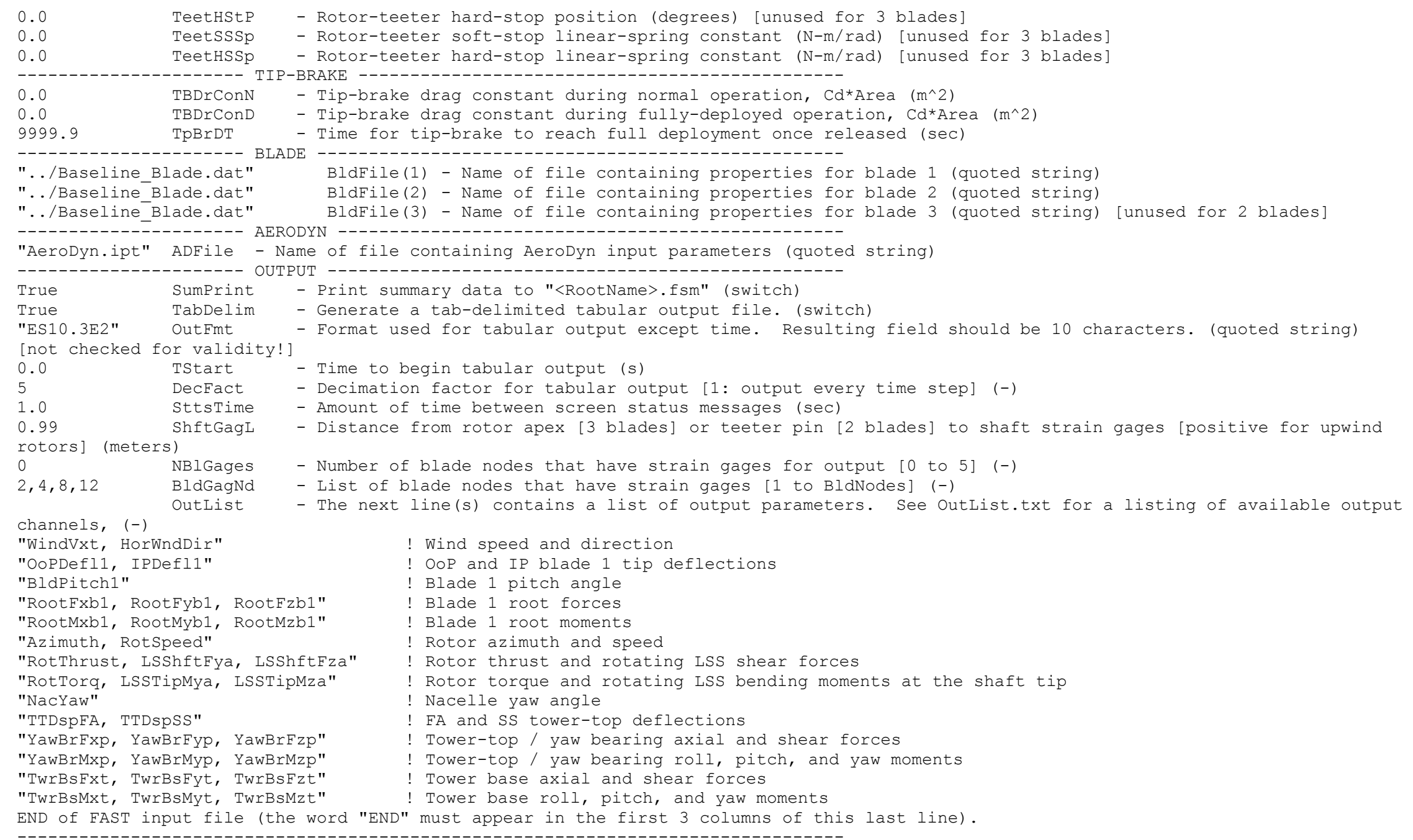




\section{FAST Blade Input File}

\begin{tabular}{|c|c|c|c|c|c|c|c|c|c|c|c|c|c|}
\hline \multirow{3}{*}{\multicolumn{14}{|c|}{$\begin{array}{l}21 \\
\text { False } \\
\text { [CURRENTLY IGNORED] (switch) }\end{array}$}} \\
\hline & & & & & & & & & & & & & \\
\hline 3.882 & & $\mathrm{Bl} d \mathrm{~F} 1 \mathrm{Dmp}(1)$ & & flap mode \#1 & structural & damping in $\mathrm{p}$ & & & & & & & \\
\hline 3.882 & & $\mathrm{Bl} d \mathrm{dF} \operatorname{\mathrm {Dmp}}(2)$ & - Blade & flap mode \#2 & structural & damping in $\mathrm{p}$ & percent of $\mathrm{cr}$ & fitical & (은) & & & & \\
\hline \multicolumn{2}{|l|}{5.900} & $\mathrm{Bl} d \mathrm{dDDmp}(1)$ & - Blade & edge mode \#1 & structural & damping in $\mathrm{p}$ & percent of $\mathrm{cr}$ & fitical & (\%) & & & & \\
\hline & & & DE ADJUST & TMENT FACTORS & -----------1 & ------------ & -------- & & & & & & \\
\hline 1.0 & & FlstTunr(1) & - Blade & flapwise moda & al stiffness & tuner, 1st & mode $(-)$ & & & & & & \\
\hline 1.0 & & FlstTunr (2) & - Blade & flapwise moda & al stiffness & tuner, 2nd & mode $(-)$ & & & & & & \\
\hline 1.0 & & AdjBlMs & - Factor & I to adjust bl & lade mass der & ensity (-) & & & & & & & \\
\hline 1.0 & & AdjFlst & - Factor & r to adjust bl & lade flap sti & iffness (-) & & & & & & & \\
\hline 1.0 & & AdjEdSt & - Factor & r to adjust bl & lade edge sti & iffness (-) & & & & & & & \\
\hline \multicolumn{14}{|c|}{ 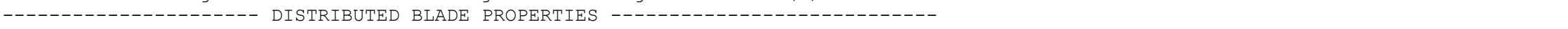 } \\
\hline $\begin{array}{l}\text { BlFract } \\
(-)\end{array}$ & $\begin{array}{l}\text { AeroCent } \\
(-)\end{array}$ & $\begin{array}{l}\text { StrcTwst } \\
(\operatorname{deg})\end{array}$ & $\begin{array}{l}\text { BmassDen } \\
(\mathrm{kg} / \mathrm{m})\end{array}$ & $\begin{array}{l}\text { FlpStff } \\
\left(\mathrm{Nm}^{\wedge} 2\right)\end{array}$ & $\begin{array}{l}\text { EdgStff } \\
\left(\mathrm{Nm}^{\wedge} 2\right)\end{array}$ & $\begin{array}{l}\text { GJStff } \\
\left(\mathrm{Nm}^{\wedge} 2\right)\end{array}$ & $\begin{array}{l}\text { EAStff } \\
(\mathrm{N})\end{array}$ & $\begin{array}{l}\text { FlpIner } \\
(\mathrm{kg} \mathrm{m})\end{array}$ & $\begin{array}{l}\text { EdgIner } \\
\left(\begin{array}{ll}\mathrm{kg} & \mathrm{m})\end{array}\right.\end{array}$ & $\begin{array}{l}\text { Flpcgof } \\
(\mathrm{m})\end{array}$ & $\begin{array}{l}\text { Edgcgof } \\
(\mathrm{m})\end{array}$ & $\begin{array}{l}\text { FlpEAOf } \\
(\mathrm{m})\end{array}$ & $\begin{array}{l}\mathrm{EdgEF} \\
(\mathrm{m})\end{array}$ \\
\hline 0.00000 & 0.250 & 11.100 & 1447.607 & $7681.46 \mathrm{E}+067$ & $7681.46 \mathrm{E}+062$ & $2655.23 \mathrm{E}+06$ & $17152.7 \mathrm{E}+06$ & 646.044 & 646.044 & 0.000 & 0.000 & 0.000 & 0.000 \\
\hline 0.02105 & 0.250 & 11.100 & 180.333 & $1169.87 \mathrm{E}+061$ & $1169.87 \mathrm{E}+06$ & $408.80 \mathrm{E}+06$ & $2640.8 \mathrm{E}+06$ & 80.480 & 80.480 & 0.000 & 0.000 & 0.000 & 0.000 \\
\hline 0.05263 & 0.250 & 11.100 & 181.672 & $1020.62 \mathrm{E}+061$ & $1092.28 \mathrm{E}+06$ & $343.81 E+06$ & $2611.3 \mathrm{E}+06$ & 68.241 & 80.113 & 0.000 & 0.032 & 0.000 & -0.005 \\
\hline 0.10526 & 0.250 & 11.100 & 183.905 & $771.88 \mathrm{E}+06$ & $962.97 \mathrm{E}+06$ & $235.50 \mathrm{E}+06$ & $2562.1 \mathrm{E}+06$ & 47.842 & 79.502 & 0.000 & 0.086 & 0.000 & 014 \\
\hline 0.15789 & 0.250 & 11.100 & 186.138 & $523.14 \mathrm{E}+06$ & $833.66 \mathrm{E}+06$ & $127.19 \mathrm{E}+06$ & $2512.9 \mathrm{E}+06$ & 27.444 & 78.892 & 0.000 & 0.140 & 0.000 & -0.023 \\
\hline 0.21053 & 0.250 & 11.100 & 188.370 & $274.40 \mathrm{E}+06$ & $704.35 \mathrm{E}+06$ & $18.87 \mathrm{E}+06$ & $2463.6 \mathrm{E}+06$ & 7.045 & 78.281 & & & 0.000 & 032 \\
\hline 0.26316 & 0.250 & 9.500 & 178.321 & $234.57 \mathrm{E}+06$ & $614.65 \mathrm{E}+06$ & $16.80 \mathrm{E}+06$ & $2332.8 \mathrm{E}+06$ & 5.963 & 68.302 & & 0 . & 0 . & 020 \\
\hline 0.31579 & 0.250 & 7.900 & 168.271 & $194.74 \mathrm{E}+06$ & $524.96 \mathrm{E}+06$ & $14.72 \mathrm{E}+06$ & $2202.0 \mathrm{E}+06$ & 4.881 & 58.323 & 0.000 & 0.182 & 0.000 & -0.007 \\
\hline 0.36842 & 0.250 & 6.300 & 158.222 & $154.90 \mathrm{E}+06$ & $435.26 \mathrm{E}+06$ & $12.64 \mathrm{E}+06$ & $2071.2 \mathrm{E}+06$ & 3.799 & 48.344 & 0.000 & 0.176 & 0.000 & 0.005 \\
\hline 0.42105 & 0.250 & 4.700 & 148.172 & $115.07 \mathrm{E}+06$ & $345.57 \mathrm{E}+06$ & $10.56 \mathrm{E}+06$ & $1940.4 \mathrm{E}+06$ & 2.717 & 66 & 0 . & 0 . & 0.000 & 0.018 \\
\hline 0.47368 & 0.250 & 3.100 & 138.123 & $75.23 \mathrm{E}+06$ & $255.87 \mathrm{E}+06$ & $8.48 \mathrm{E}+06$ & $1809.6 \mathrm{E}+06$ & 1.635 & 28.387 & 0.000 & 0.164 & 0.000 & 0.030 \\
\hline 0.52632 & 0.250 & 2.600 & 122.896 & $62.49 \mathrm{E}+06$ & $217.87 \mathrm{E}+06$ & $7.12 \mathrm{E}+06$ & $1605.3 \mathrm{E}+06$ & 1.367 & 24.050 & 0.000 & 0.168 & 0.000 & 0.038 \\
\hline 0.57895 & 0.250 & 2.100 & 107.669 & $49.75 \mathrm{E}+06$ & $179.86 \mathrm{E}+06$ & $5.76 \mathrm{E}+06$ & $1401.1 \mathrm{E}+06$ & 1.099 & 19.714 & & & & 0.047 \\
\hline 0.63158 & 0.250 & 1.600 & 92.442 & $37.01 \mathrm{E}+06$ & $141.86 \mathrm{E}+06$ & $4.40 \mathrm{E}+06$ & $1196.8 \mathrm{E}+06$ & 0.831 & 15.377 & 0.000 & 0.176 & 0.000 & 0.055 \\
\hline 0.68421 & 0.250 & 1.100 & 77.215 & $24.27 \mathrm{E}+06$ & $103.85 \mathrm{E}+06$ & $3.04 \mathrm{E}+06$ & $992.6 \mathrm{E}+06$ & 0.564 & 11.041 & 0.000 & 0.179 & 0.000 & 0.063 \\
\hline 0.73684 & 0.250 & 0.600 & 61.988 & $11.53 \mathrm{E}+06$ & $65.85 E+06$ & $1.68 \mathrm{E}+06$ & $788.3 E+06$ & 0.296 & 6.704 & 0.000 & 0.183 & 0.000 & 0.071 \\
\hline 0.78947 & 0.250 & 0.480 & 51.861 & $9.27 \mathrm{E}+06$ & $54.25 E+06$ & $1.38 \mathrm{E}+06$ & $654.3 E+06$ & 0.240 & 5.513 & 0 . & 0.1 & 0.000 & 0.077 \\
\hline 0.84211 & 0.250 & 0.360 & 41.734 & $7.01 \mathrm{E}+06$ & $42.66 \mathrm{E}+06$ & $1.08 \mathrm{E}+06$ & $520.4 \mathrm{E}+06$ & 0.185 & 4.322 & 0.000 & 0.198 & 0.000 & 0.082 \\
\hline 0.89474 & 0.250 & 0.240 & 31.607 & $4.75 E+06$ & $31.06 \mathrm{E}+06$ & $0.78 \mathrm{E}+06$ & $386.4 \mathrm{E}+06$ & 0.130 & 3.130 & 0.0 & & & 0.087 \\
\hline 0.94737 & 0.250 & 0.120 & 21.480 & $2.49 E+06$ & $19.47 \mathrm{E}+06$ & $0.48 \mathrm{E}+06$ & $252.4 \mathrm{E}+06$ & 0.074 & 1.939 & 0.0 & 0.212 & 0. & 0.092 \\
\hline 1.00000 & 0.250 & 0.000 & 11.353 & $0.23 E+06$ & $7.87 \mathrm{E}+06$ & $0.18 \mathrm{E}+06$ & $118.5 \mathrm{E}+06$ & 0.019 & 0.747 & 0.000 & 0.220 & 0.000 & 0.098 \\
\hline \multirow{2}{*}{\multicolumn{14}{|c|}{ BldFliSh (2) - Flap, }} \\
\hline & & & & & & & & & & & & & \\
\hline \multicolumn{2}{|l|}{1.6525} & BldFl1Sh (3) & - & coeff of $x^{\wedge} 3$ & & & & & & & & & \\
\hline \multicolumn{2}{|l|}{-1.5682} & BldFl1Sh (4) & - & coeff of $x^{\wedge} 4$ & & & & & & & & & \\
\hline \multirow{2}{*}{\multicolumn{2}{|c|}{$\begin{array}{r}1.6947 \\
-0.8628\end{array}$}} & BldFl1Sh (5) & - & coeff of $x^{\wedge} 5$ & & & & & & & & & \\
\hline & & BldFl1Sh (6) & - & coeff of $x^{\wedge} 6$ & & & & & & & & & \\
\hline \multicolumn{2}{|l|}{-0.3008} & BldF12Sh (2) & - Flap, & coeff of $x^{\wedge} 2$ & & & & & & & & & \\
\hline \multirow{2}{*}{\multicolumn{2}{|c|}{$\begin{array}{l}-1.9968 \\
-4.6564\end{array}$}} & BldFl2Sh (3) & - & coeff of $x^{\wedge} 3$ & & & & & & & & & \\
\hline & & BldF12Sh (4) & - & coeff of $x^{\wedge} 4$ & & & & & & & & & \\
\hline
\end{tabular}


16.9661

$-9.0121$

0.3165

3.2618

$-6.4005$

6.0367

$-2.2146$

BldFl2Sh(5) - , coeff of $x^{\wedge} 5$

BldFl2Sh(6) - , coeff of $x^{\wedge} 6$

BldEdgSh (2) - Edge', coeff of $x^{\wedge} 2$

BldEdgSh (3) - , coeff of $x^{\wedge} 3$

$\begin{array}{ll}\text { BldEdgSh (3) - } & \text {, Coeff of } x^{\wedge} 3 \\ \text { BldEdgSh (4) - } & \text {, coeff of } x^{\wedge} 4\end{array}$

$\begin{array}{ll}\text { BldEdgSh (4) - } & \text {, Coeff of } x^{\wedge} 4 \\ \text { BldEdgSh (5) - } & \text {, coeff of } x^{\wedge} 5\end{array}$

BldEdgSh (6) - , coeff of $x^{\wedge} 6$ 
FAST Tower Input File

FAST TOWER FILE

1.5 MW baseline tower model properties from "InputDatal.5A08V07adm.xls" (from C. Hansen) with bugs removed.

NTwInpSt - NOWER PARAMETERS -- Numbr of input stations to specify tower geometry

False CalcTMode - Calculate tower mode shapes internally $\{\mathrm{T}$ : ignore mode shapes from below, F: use mode shapes from below\} [CURRENTLY IGNORED] (switch)

[CURRENTLY IGNORED] (SWitch) - TOwer 1st fore-aft mode structural damping ratio (8)

3.435 TwrFADmp (1) - Tower 1st fore-aft mode structural damping ratio (\%)

$\begin{array}{ll}3.435 & \text { TwrFADmp (2) - Tower 2nd fore-aft mode structural damping ratio (\%) } \\ 3.435 & \text { TwrSSDmp (1) - Tower lst side-to-side mode structural damping ratio (\%) }\end{array}$

3.435 TwrSSDmp (2) - Tower 2nd side-to-side mode structural damping ratio (\%)

1.0 FAStTunr(1) - Tower fore-aft modal stiffness tuner, 1st mode (-)

1.0 FAStTunr(2) - Tower fore-aft modal stiffness tuner, 2nd mode (-)

1.0 SSStTunr(1) - Tower side-to-side stiffness tuner, 1st mode (-)

1.0 SSStTunr(2) - Tower side-to-side stiffness tuner, 2nd mode (-)

1.0 AdjTwMa - Factor to adjust tower mass density (-)

$\begin{array}{lllll}1.0 & \text { AdjFASt } & \text { - Factor to adjust tower fore-aft stiffness (-) } \\ 1.0 & \text { AdjSSSt } & \text { - Factor to adjust tower side-to-side stiffness (-) }\end{array}$ ------------------ DISTRIBUTED TOWER PROPERTIES

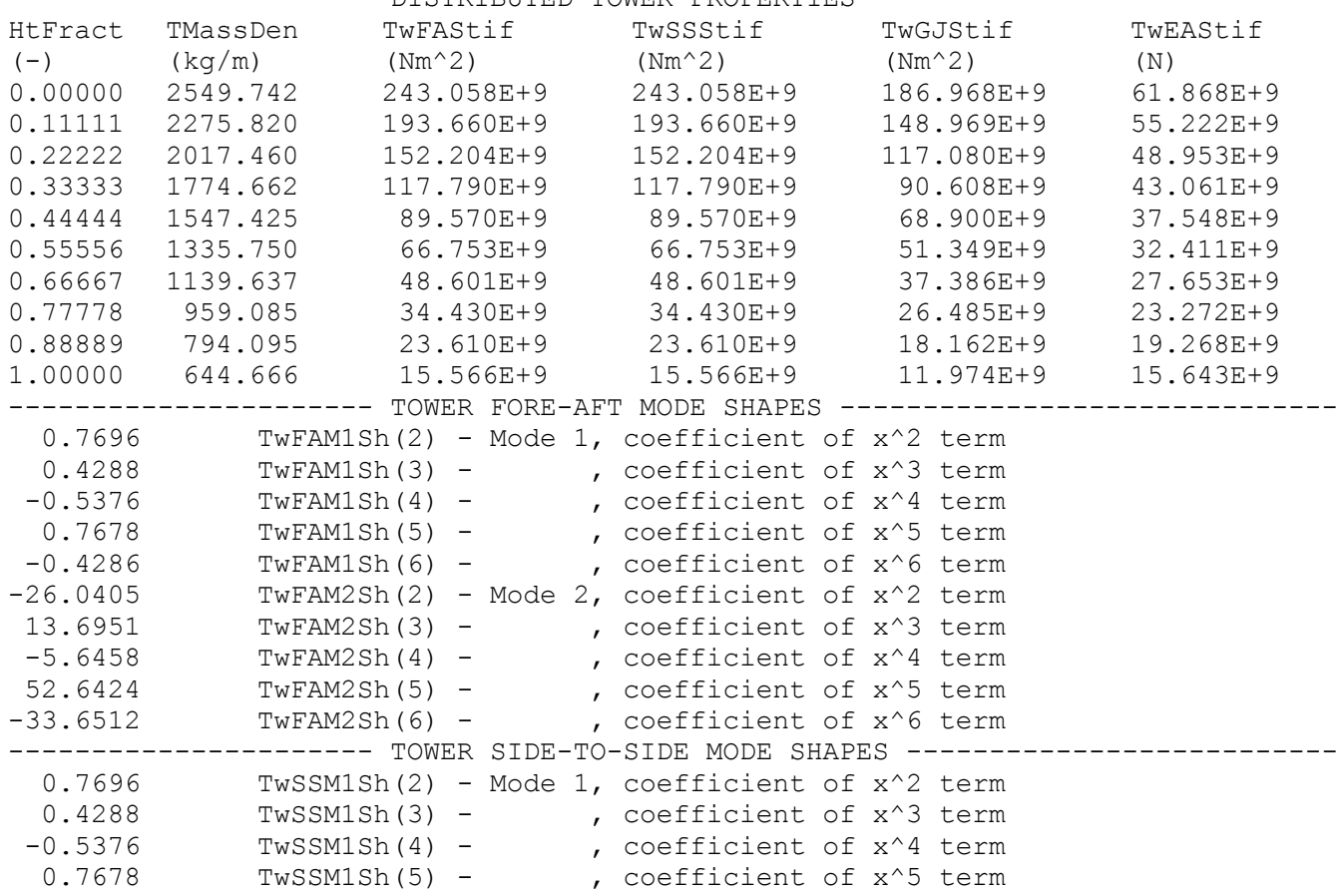

$\begin{array}{cccl}\begin{array}{c}\text { TwFAIner } \\ (\mathrm{kg} \mathrm{m})\end{array} & \begin{array}{c}\text { TwSSIner } \\ (\mathrm{kg} \mathrm{m})\end{array} & \begin{array}{c}\text { TwFAcgOf } \\ (\mathrm{m})\end{array} & \begin{array}{l}\text { TwSScgOf } \\ (\mathrm{m})\end{array} \\ 9540.03 & 9540.03 & 0.0 & 0.0 \\ 7601.16 & 7601.16 & 0.0 & 0.0 \\ 5974.02 & 5974.02 & 0.0 & 0.0 \\ 4623.25 & 4623.25 & 0.0 & 0.0 \\ 3515.63 & 3515.63 & 0.0 & 0.0 \\ 2620.07 & 2620.07 & 0.0 & 0.0 \\ 1907.59 & 1907.59 & 0.0 & 0.0 \\ 1351.37 & 1351.37 & 0.0 & 0.0 \\ 926.69 & 926.69 & 0.0 & 0.0 \\ 610.96 & 610.96 & 0.0 & 0.0\end{array}$


TwSSM1Sh(6) - , coefficient of $x^{\wedge} 6$ term TwSSM2Sh(2) - Mode 2, coefficient of $x^{\wedge} 2$ term TwSSM2Sh(3) - , coefficient of $x^{\wedge} 3$ term

TwSSM2Sh(4) - , coefficient of $x^{\wedge} 4$ term

$\begin{array}{llll}\text { TwSSM2Sh(4) - } & \text {, coefficient of } x^{\wedge} 4 \text { term } \\ \text { TwSSM2Sh(5) - } & , \text { coefficient of } x^{\wedge} 5 \text { term }\end{array}$

TwSSM2Sh $(6)$, coefficient of $x^{\wedge} 6$ term 


\section{FAST AeroDyn Input File}

1.5 MW baseline aerodynamic parameters for FAST. BEDDOES

NO_CM

Sysunits - System of units for used for input and output [must be SI for FAST] (unquoted string)

DYN̄IN

StallMod - Dynamic stall included [BEDDOES or STEADY] (unquoted string)

SWIRL IndModel - Induction-factor model [NONE or WAKE or SWIRL] (unquoted string)

0.005 AToler - Induction-factor tolerance (convergence criteria) (-)

PRANDtl TLModel - Tip-loss model (EQUIL only) [PRANDt1, GTECH, or NONE] (unquoted string)

PRANDtl HLModel - Hub-loss model (EQUIL only) [PRANdtl or NONE] (unquoted string)

"../../Wind/NoShr_12.wnd" WindFile - Name of file containing wind data (quoted string)

$84.2876 \quad \mathrm{HH}^{-}$- Wind reference (hub) height [TowerHt+Twr2Shft+OverHang*SIN(NacTilt)] (m)

$0.0 \quad$ TwrShad - Tower-shadow velocity deficit (-)

9999.9 ShadHWid - Tower-shadow half width (m)

9999.9 T_Shad_Refpt - Tower-shadow reference point (m)

1.225 Rho - Air density ( $\mathrm{kg} / \mathrm{m}^{\wedge} 3$ )

1.4639e-5 KinVisc - Kinematic air viscosity [CURRENTLY IGNORED] (m^2/sec)

0.004 DTAero - Time interval for aerodynamic calculations (sec)

$4 \quad$ Numfoil - Number of airfoil files (-)

"...../AeroData \cylinder.dat" FoilNm - Names of the airfoil files [NumFoil lines] (quoted strings)

"..././AeroData \s818 2703.dat"

.....AeroData $\backslash$ s825_2103.dat"

15 BldNodes - Number of blade nodes used for analysis (-)

RNodes

2.85833

5.07500

7.29167

AeroTwst DRNodes

Chord

TFOil PrnElm

9.50833

11.10

2.21667

1.949

11.10

2.21667

2.269

10.41

2.21667

2.589

2.743
2.578
2.412

13.94167

16.15833

18.37500

8.38

2. 21667

2. 578

6.35
4.33

2.21667

2.412
2.247

.85

20.59167

22.80833

25.02500

27.24167

29.45833

31.67500

2.21667

2.21667

2.21667

1.58

0.95

2. 21667

2. 21667

2.21667

0.38

33.89167

2.21667

2.082

1.916

1.751

1.585

1.427

1.278

0.23

$\begin{array}{lll}0.08 & 2.21667 & 0.980\end{array}$

PRINT

PRINT

PRINT

PRINT

PRINT

PRINT

PRINT

PRINT

PRINT

PRINT

PRINT

PRINT

PRINT 


\section{RCAS Input File for the Turbine Spinning in a Vacuum}

! RCAS model of 1.5 MW, 3-bladed, upwind, turbine used for RCAS validation.

Model properties from "InputDatal.5A08V07adm.xls" (from C. Hansen) with bugs removed.

\begin{tabular}{|c|c|c|}
\hline$\star \star \star *$ & Wind Turbine Baseline Model & $\star \star \star *$ \\
\hline *** & Elastic Tower & * \\
\hline 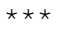 & Linear Spring Driveshaft & * \\
\hline & 3 Elastic Blades & ^ \\
\hline & Rigid hub & $\star \star$ \\
\hline & 0 deg Precone & ** \\
\hline & No Aero & $x$ \\
\hline & SI Units & ^ \\
\hline
\end{tabular}

MENU RCASROOT

Reinitialize/Clean RDB

Initialize/Load screen

! Return to command mode

COMMAND

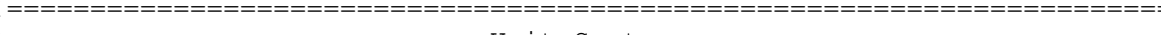

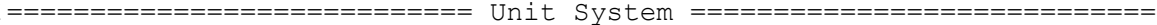

$!===============$

S UNITSYSTEM

Unity System Name

ENGLISH, SI

!

! $============================$ MODEL

S SUBSYSIDS

List subsystem IDs which must be unique; one ID per row.

A towerss

! tower and nacelle

rotorss

S GFRAMEORIG

G frame origin of the node to which the G frame is attached.
Subsystem
Primitive
Name
Structure
Name
Node
Translational Rotational
$X$ Y Z
$X$ Y Z

G frame is attached. 


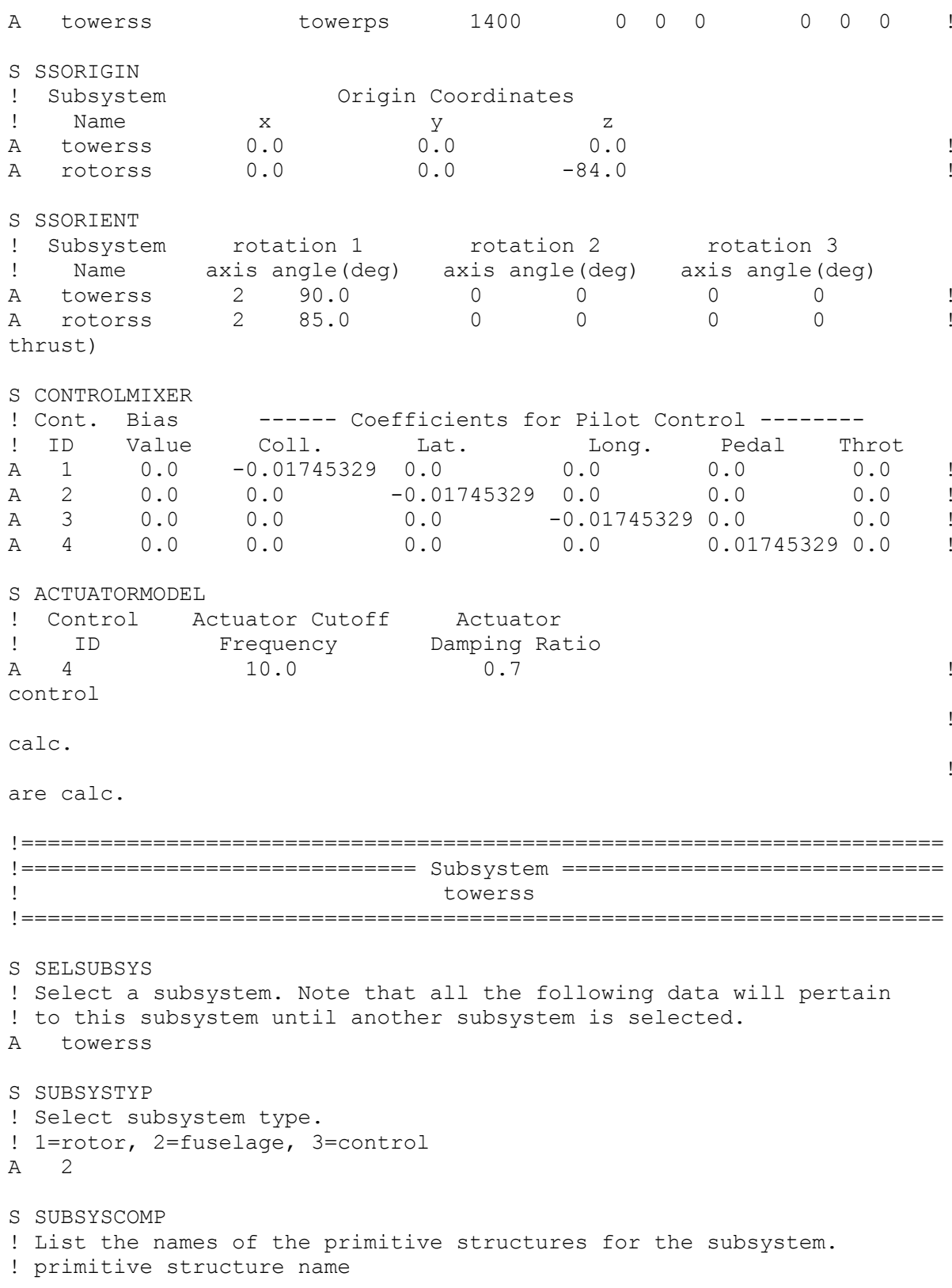




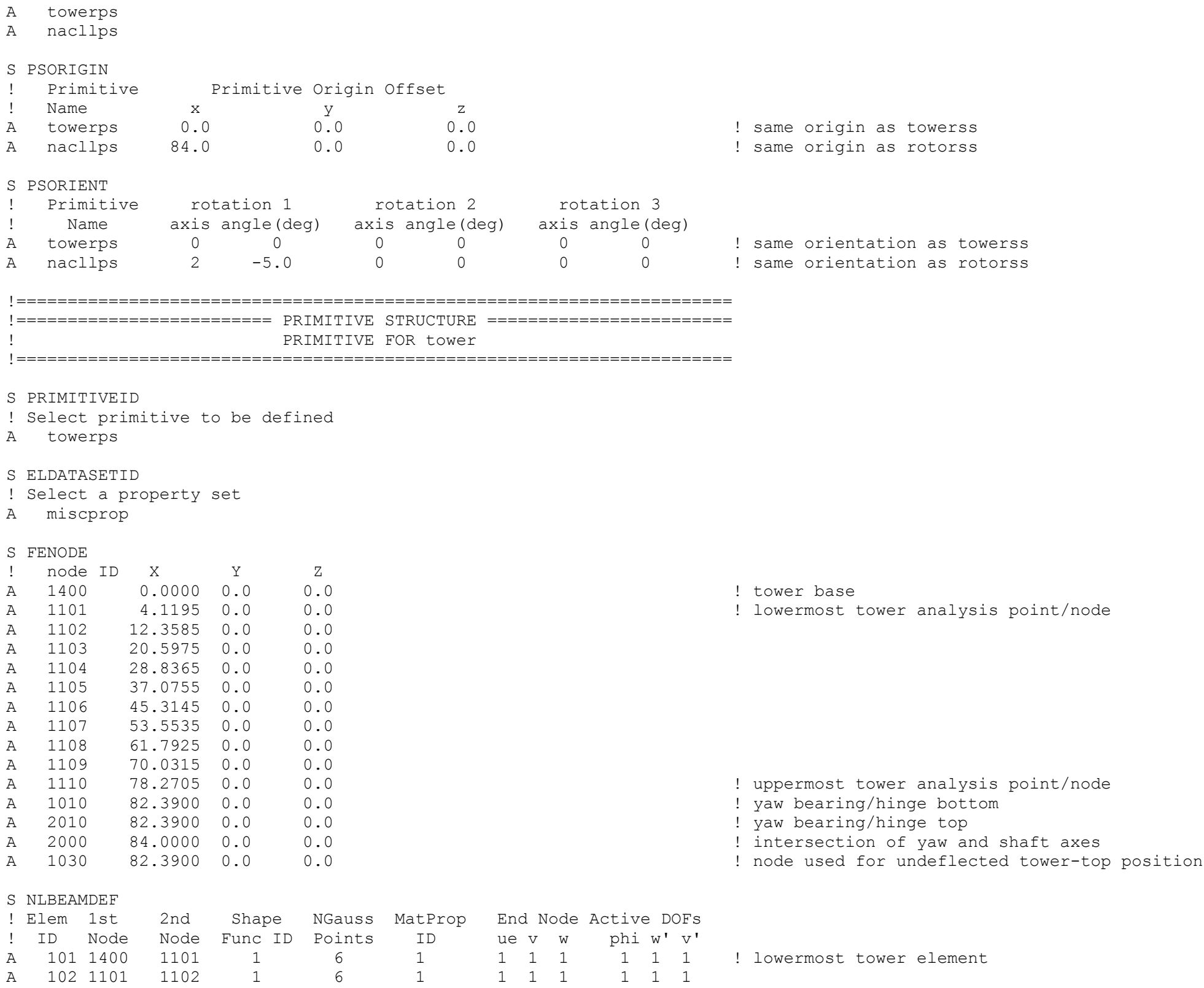




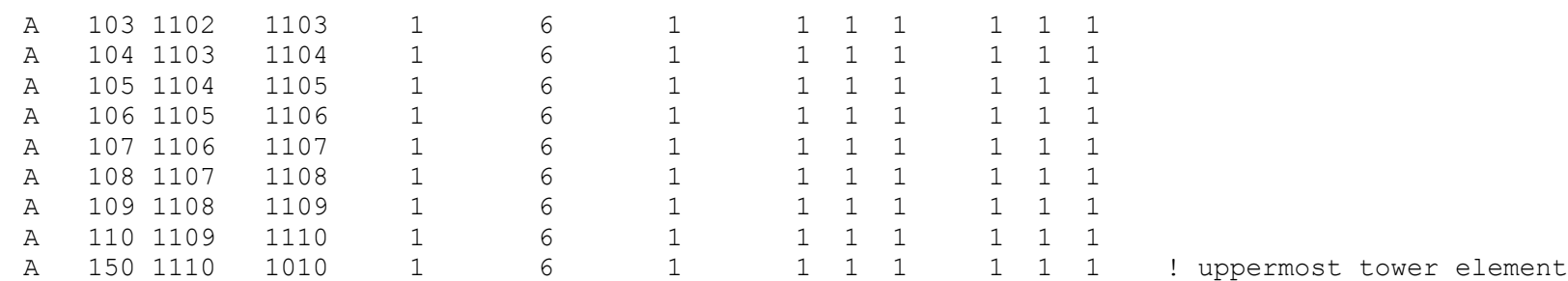

! Structural properties may be entered here, or in a table in next screen

Structural twist is defined relative to the E frame

PRP-INDEX, ELID, PRP-LOC, PRPID, STR-TWIST

! Specify the structural property data table (file)

$\begin{array}{lll}\text { !Element } & \text { Refernce } & \text { Property } \\ \text { ! ID } & \text { origin } & \text { Filename }\end{array}$

ID Origi $101 \quad 0.0$

. /BASELINE_TOWER.TAB
. /BASELINE_TOWER.TAB

../BASELINE_TOWER.TAB

. . /BASELINE_TOWER.TAB

. . /BASELINE TOWER. TAB

. . BASELINE TOWER. TAB

. /BASELINE TOWER. TAB

/BASEITNE TOWFR TAB

. BASELINE TOWER. TAB

. BASELINE TOWER. TAB

. /BASELINE_TOWER.TAB

\begin{tabular}{|c|c|c|}
\hline & 0.0 & . ./BASELINE ${ }^{-}$TOWER.TAB \\
\hline & 0.0 & ../BASELINE_TOWER.TA \\
\hline 11 & 0.0 & . /BASELINE ${ }^{-}$TOWER \\
\hline & 0.0 & LINE \\
\hline
\end{tabular}

S PSMODALDAMP

!row index

mode number Damping ratio

1:9999

3. $435 \mathrm{E}-02$

S RIGIDBAR

$\begin{array}{ccccccc}\text { ! } & \text { Element } & \text { Node1 } & \text { Node2 } & \text { Center of gravity offse } \\ ! & \text { ID } & \text { ID } & \text { ID } & \text { X } & \text { Y } & \text { Z } \\ \text { A } & 2100 & 2010 & 2000 & 0.0 & 0.0 & 0.0 \\ \text { A } & 1030 & 1400 & 1030 & 0.0 & 0.0 & 0.0\end{array}$

! bed-plate

! link from tower base to undeflected tower-top

N

$\begin{array}{ccccccccc}\text { ! } & \text { Element } & \text { Element } & & & & & \text { Inertia } & \text { Terms } \\ ! & \text { ID } & \text { Mass } & \text { Ixx } & \text { Ixy } & \text { Ixz } & \text { Iyy } & \text { Iyz } & \text { Izz } \\ \text { A } & 2100 & 0.0 & 0.0 & 0.0 & 0.0 & 0.0 & 0.0 & 0.0 \\ \text { A } & 1030 & 0.0 & 0.0 & 0.0 & 0.0 & 0.0 & 0.0 & 0.0\end{array}$

S HINGE

$\begin{array}{cccccccc}\text { ! Elem. } & \text { Node1 } & \text { Node2 } & \text { Hinge } & \text { Free or } & \text { Spring } & \text { Damper } \\ \text { ! } & \text { ID } & \text { ID } & \text { ID } & \text { Type } & \text { Controlled } & \text { Constant } & \text { Constant } \\ \text { A } & 2010 & 1010 & 2010 & \text { P } & 1 & 0.0 & 0.0\end{array}$
0.0
0.0
! yaw bearing/hinge 


$\begin{array}{lccccc}\text { S CONTROLCONNECT } & & & \\ \text { ! Control } & \text { Swashplate } & \text { Swashplate } & \text { Element Type } & \text { Element } \\ ! & \text { ID } & \text { or Direct } & \text { Phase (deg) } & (\text { HIN/AUX/ENG ...) } & \text { or ACP ID } \\ \text { A } & 4 & \text { DIRECT } & 0.0 & \text { HIN } & 2010\end{array}$

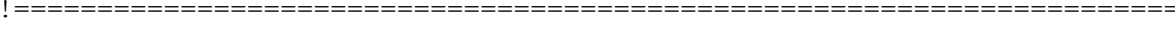

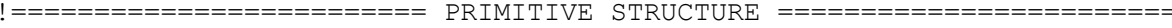

!

PRIMITIVE FOR nacelle

\section{S PRIMITIVEID}

! Select primitive to be defined

A naclips

S ELDATASETID

! Select a property set

A miscprop

S FENODE

$\begin{array}{ccccc}\text { ! } & \text { node ID } & X & Y & Z \\ \text { A } & 2130 & 0.0000 & 0.0 & 0.0\end{array}$

A $2001 \quad 1.0000 \quad 0.0 \quad 0.0$

! intersection of yaw and shaft axes

! one unit above along local x-axis

S RIGIDBAR

Element Node1 Node2 Center of gravity offset

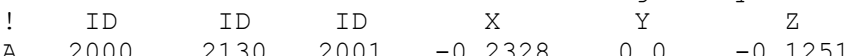

A $2000-2130=2001-0.2328=0.0=-0.1251$

! nacelle CM

Element Element Inertia Terms

ID Mass Ixx Ixy Ixz Iyy Iyz Izz

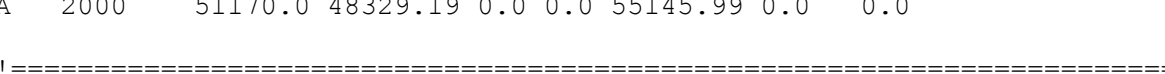

! $======0==0=0$

S CONNCONST

! constraint ID, DOFL( PS name, node ID ), DOFR( PS name, node ID)

A 1 nacllps 2130 towerps 2000

! connect tower and nacelle PSs together at node 2000

(this effectively eliminates node 2130 from the model)

!

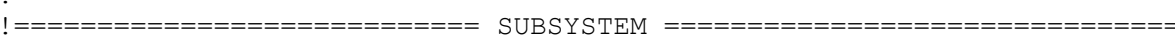

! $\quad$ ROTOR

S SELSUBSYS

! Select a subsystem. Note that all the following data will pertain

! to this subsystem until another subsystem is selected. 


\section{S SUBSYSTYP}

! Select subsystem type.

! 1=rotor, 2=fuselage, 3=control

A 1

S SUBSYSCOMP

! List the names of the primitive structures for the subsystem. Primitive Structure

Name

Isshetps

A bladelps

A blade2ps

A blade3ps

\section{S CORNODE}

! identify center node for the rotor subsystem

! Prim_str_ID Node ID

\begin{tabular}{|c|c|c|c|c|c|c|c|}
\hline \multicolumn{8}{|c|}{ S BLADECOMP } \\
\hline Blade & & & & structure & Name (s) & & \\
\hline Index & 1 & 2 & 3 & 4 & 5 & 6 & 7 \\
\hline 1 & bladelps & -- & -- & -- & -- & -- & -- \\
\hline 2 & blade2ps & -- & -- & -- & -- & -- & -1 \\
\hline 3 & blade3ps & -- & -- & -- & -- & -- & -1 \\
\hline
\end{tabular}

S PSORIGIN

! Primitive $\quad$ Primitive Origin Offset

$\begin{array}{clccc}! & \text { Name } & x & y & z \\ \text { A } & \text { lsshftps } & 0.0 & 0.0 & 0.0 \\ \text { A } & \text { bladelps } & 0.0 & 0.0 & -3.3 \\ \text { A } & \text { blade2ps } & 0.0 & 0.0 & -3.3 \\ \text { A } & \text { blade3ps } & 0.0 & 0.0 & -3.3\end{array}$

\section{0 \\ $-3.3$}

$-3.3$

rotation 2

rotation 3

S PSORIENT

! Primitiv

! Name axis angle(deg)

A lsshftps 3

bladelps 3

blade2ps 0.0

Alade 3 s

120.0 axis angle (deg)

0 $\operatorname{axis}$
0

0
0

S ROTORPARAM

! Rotor Rotational Speed ( $\mathrm{rad} / \mathrm{sec}$ )

A 2.14288

!

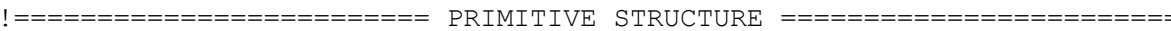

PRIMITIVE FOR low speed shaft

! intersection of yaw and shaft axes

! hub center

hub center

! hub center 


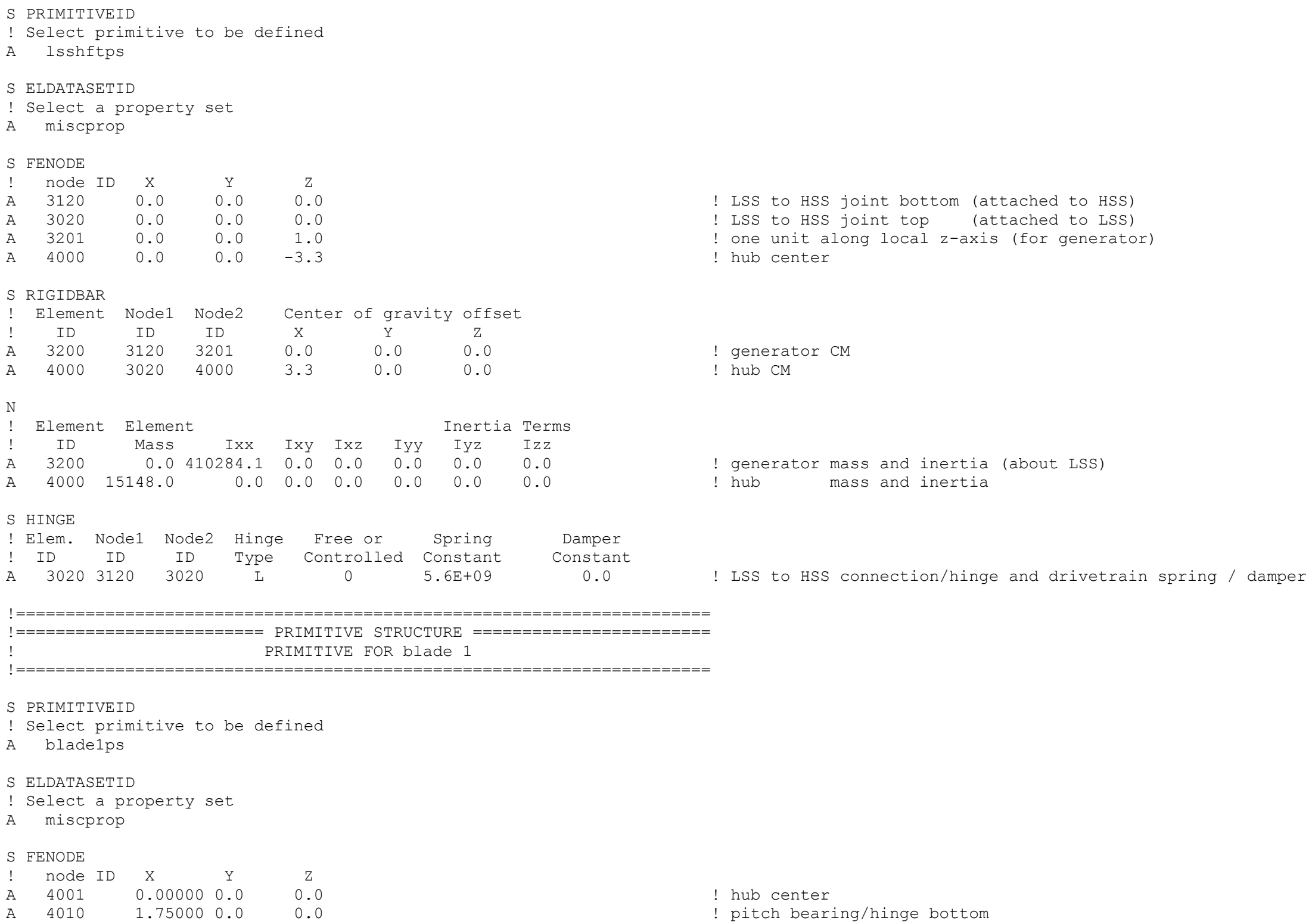

! LSS to HSS joint bottom (attached to HSS)

! LSS to HSS joint top (attached to LSS)

! one unit along local z-axis (for generator)

! hub center

generator mass and inertia (about LSS)

! hub mass and inertia

! LSS to HSS connection/hinge and drivetrain spring / damper 


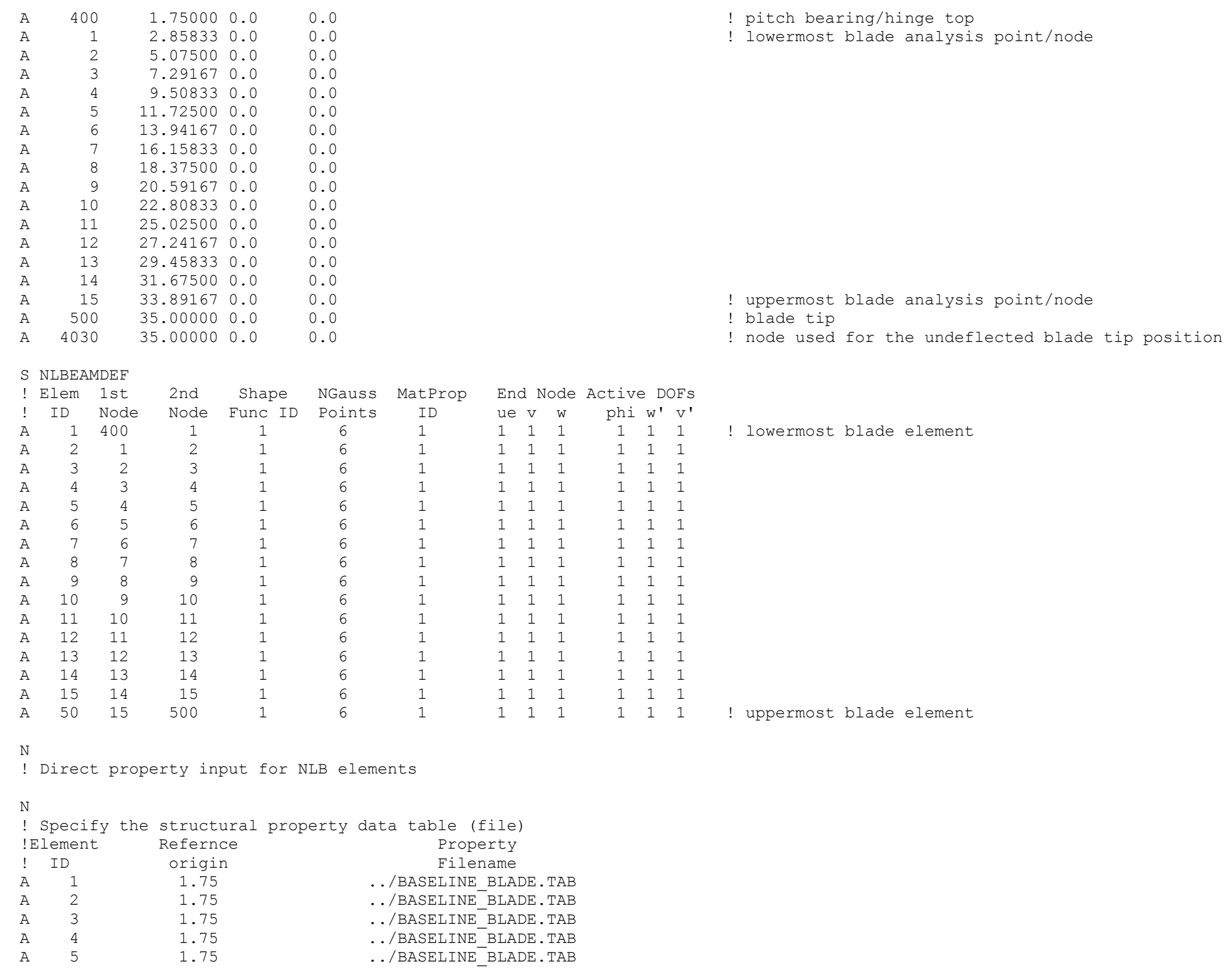




\begin{tabular}{|c|c|c|c|}
\hline A & 6 & 1.75 & . /BASELINE BLADE.TAB \\
\hline A & 7 & 1.75 & . . BASELINE_BLADE.TAB \\
\hline A & 8 & 1.75 & . . BASELINE BLADE.TAB \\
\hline A & 9 & 1.75 & . . BASELINE_BLADE.TAB \\
\hline A & 10 & 1.75 & . . /BASELINE_BLADE.TAB \\
\hline A & 11 & 1.75 & . /BASELINE_BLADE.TAB \\
\hline A & 12 & 1.75 & . . BASELINE_BLADE.TAB \\
\hline A & 13 & 1.75 & . . BASELINE_BLADE.TAB \\
\hline A & 14 & 1.75 & . . BASELINE_BLADE.TAB \\
\hline A & 15 & 1.75 & . . BASELINE_BLADE.TAB \\
\hline A & 50 & 1.75 & . / BASELINE BLADE.TAB \\
\hline
\end{tabular}

S PSMODALDAMP

!row index

A 1

DOFS)

A 2

A 3

$\begin{array}{ll}\text { A } & 4 \\ A & 5\end{array}$

A 6

$\begin{array}{ll}\text { A } & 7 \\ \text { A } & 8\end{array}$

$\begin{array}{lr}\text { A } & 9 \\ \text { A } & 10\end{array}$

A 11

S RIGIDBAR

\begin{tabular}{|c|c|c|c|c|c|}
\hline Element & Node1 & Node2 & Center & of gravity & offse \\
\hline ID & ID & ID & $\mathrm{x}$ & $\mathrm{Y}$ & $\mathrm{z}$ \\
\hline 4020 & 4001 & 4010 & 0.0 & 0.0 & 0.0 \\
\hline 030 & 4001 & 30 & 0.0 & 0 & 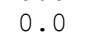 \\
\hline
\end{tabular}

mode number Damping ratio

3. $882 \mathrm{E}-02$

$5.900 \mathrm{E}-02$

$3.882 \mathrm{E}-02$

$5.900 \mathrm{E}-02$

$3.882 \mathrm{E}-02$

DE-02

$3.882 \mathrm{E}-02$

$3.882 \mathrm{E}-02$

$1.000 \mathrm{E}-02$

$3.882 \mathrm{E}-02$

$1.000 \mathrm{E}-02$

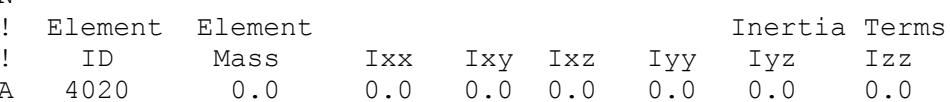

$\begin{array}{llllllll}\text { A } 4030 & 0.0 & 0.0 & 0.0 & 0.0 & 0.0 & 0.0 & 0.0\end{array}$

S HINGE

! Elem. Node1 Node2 Hinge Free or Spring Damper

! ID ID ID Type Controlled Constant Constant
1
0.0
0.0

S CONTROLCONNECT
! Contro
Swashplate
Swashplate
Element Type
Element
! ID
or Direct
Phase (deg) (HIN/AUX/ENG ...)
$\mathrm{HIN}$
or ACP ID
100

! blade edge mode (")

! blade flap mode (")

! blade edge mode (")

! blade flap mode (")

! blade torional mode (")

! blade flap mode (")

! blade flap mode (")

blade torsional mode (")

! blade flap mode (")

! all other blade modes

! portion of hub for blade 1

! link from hub to undeflected tip of blade 1

le="

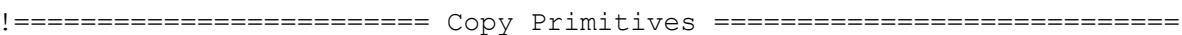

! pitch bearing/hinge 

S PRIMIT

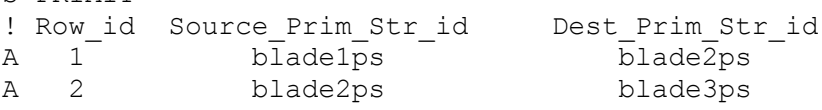

EXIT

COMMAND

copyprimstruct

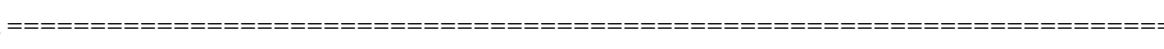
$\begin{aligned} !===================== & \text { CONTROL CONNECTIONS } \\ & \text { FOR blade } 1 \text { and blade }\end{aligned}$

S PRIMITIVEID

! Select primitive to be defined

C blade2ps

S CONTROLCONNECT

$\begin{array}{ccccc}\text { Control } & \text { Swashplate } & \text { Swashplate } & \text { Element Type } & \text { Element } \\ \text { ID } & \text { or Direct } & \text { Phase (deg) } & \text { (HIN/AUX/ENG ...) } & \text { or ACP ID }\end{array}$

! ID

or Direct

Phase (deg)

HIN/AUX/ENG ...

A 2

DIRECT

0.0

HIN

100

PRIMITIVEID

! Select primitive to be defined

C blade3ps

S CONTROLCONNECT

\begin{tabular}{|c|c|c|c|c|}
\hline Control & Swashplate & Swashplate & Element Type & Element \\
\hline ID & or Direct & Phase (deg) & $(\mathrm{HIN} / \mathrm{AUX} / \mathrm{ENG} \ldots)$ & or ACP ID \\
\hline 3 & DIRECT & 0.0 & HIN & 100 \\
\hline
\end{tabular}
A 3
DIRECT
100

!

! $========================$ Connect Primitives $========================0$

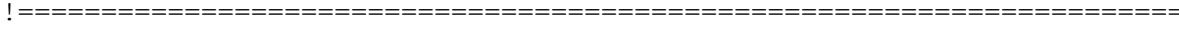

S CONNCONST

! constraint ID, DOFL( PS name, node ID), DOFR( PS name, node ID)

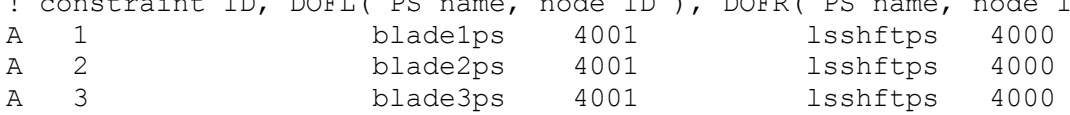

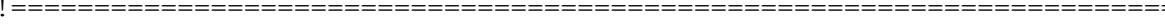

! $=========================$ Connect Subsystem $=========================$.

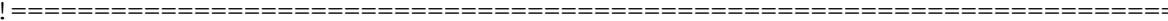

! connect lss and blade PSs together at node 4000

(this effectively eliminates node(s) 4001 from the model) 


\begin{tabular}{|c|c|c|c|c|c|c|}
\hline \multicolumn{4}{|c|}{ Non-rotating } & \multicolumn{3}{|c|}{ Rotating } \\
\hline Cnstr. & Subsystem & Primitive & Node & Subsystem & Primitive & \\
\hline ID & Name & Name & ID & Name & Name & \\
\hline 1 & towerss & towerps & 2000 & rotorss & Isshftps & 12 \\
\hline
\end{tabular}

! connect rotating and non-rotating subsystems

at intersection of yaw and shaft axes

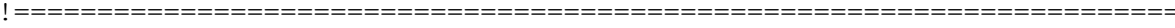
! $======================$ MISCELLANEOUS PROPERTIES $======================$

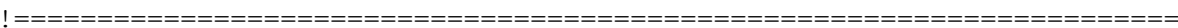

S ELEPROPID

! List the names of element property data sets.

! element_prop_ID

A miscprop

S NLBSHAPE
NLB Shape Function
$\begin{array}{llr}---- \text { Shape } & \text { Function Orders } \\ \text { Axial } & \text { Bending } & \text { Torsion } \\ & \end{array}$
Set ID
Axial
A 1
0
1

! linear for axial and torsional modes, default for bending

(therefore, the NLB is effectively a linear beam model)

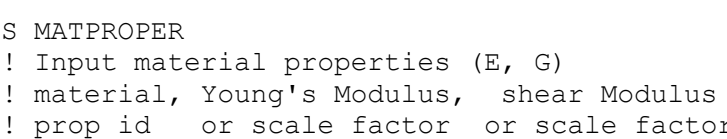

Case_id Case Title

A $01^{-}$maneuver

S INITCOND

! Initial Pilot Controls

! collective, lateral, longitudinal, pedal, throttle 


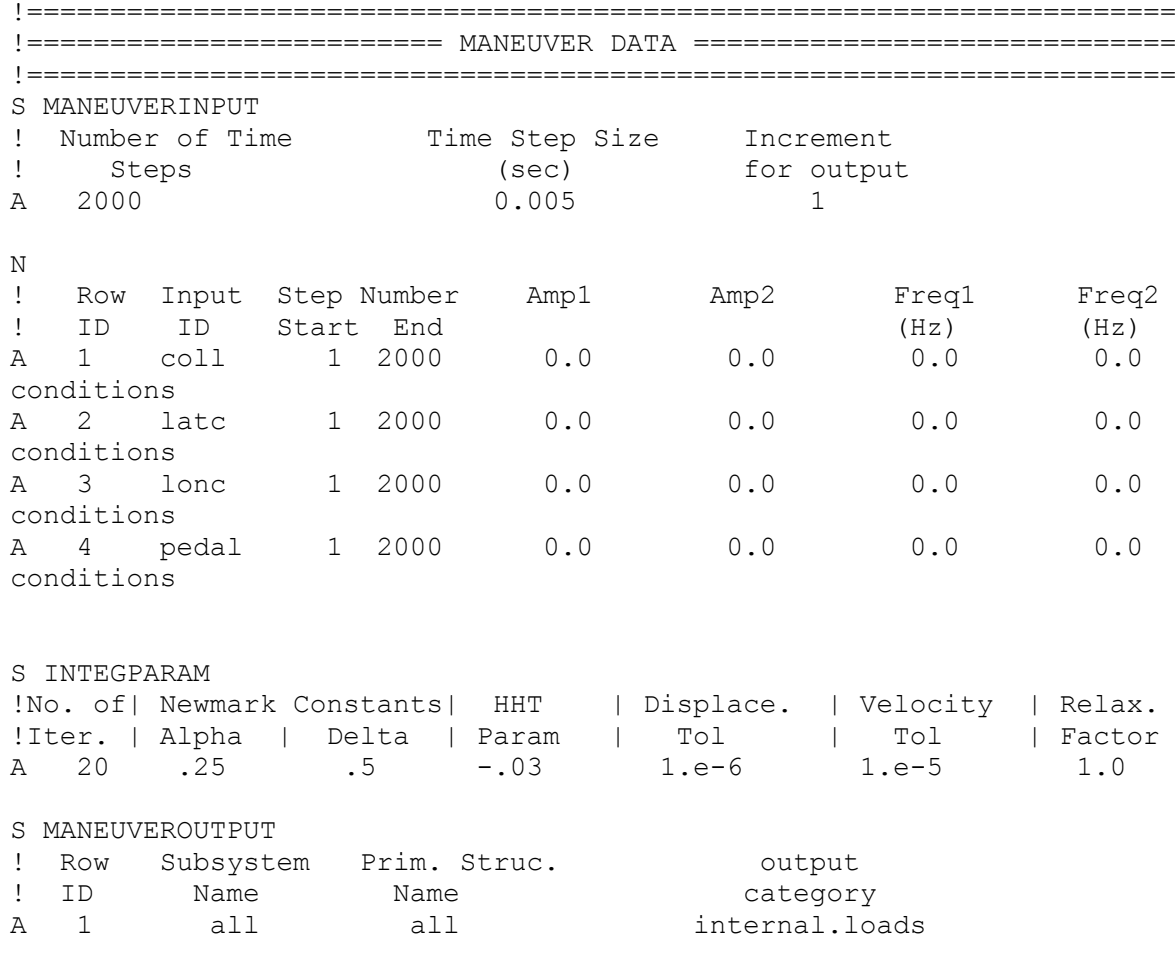

!

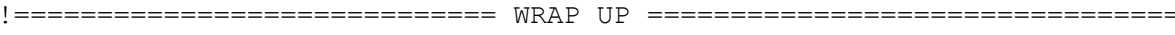

S RUNALLCASES

! Run All Cases Flag $(0 / 1)$

A 0

EXIT

COMMAND 
MENU RUNANALYSIS 


\section{RCAS Blade Properties Input File}

! 1.5 MW baseline blade model properties from "InputDatal.5A08V07adm.xls" (from C. Hansen) with bugs removed.

NOTE: "! CatName" is a keyword telling RCAS that the next set of data to be read in is data for category "CatName".

Thus, the order of fields in this file is unimportant.

Reference/flexible length of beam (m)

IM REFLENGTH

33.25

! Structural twist about X (rad)

!M BSTRUCTW

$0.00000-0.19373$

$0.02105-0.19373$

$0.05263-0.19373$

$0.10526-0.19373$

$0.15789-0.19373$

$0.21053-0.19373$

$0.26316-0.16581$

$0.31579-0.13788$

$0.36842-0.10996$

$0.42105-0.08203$

$0.47368-0.05411$

$0.52632-0.04538$

$0.57895-0.03665$

$0.63158-0.02793$

$0.68421-0.01920$

$0.73684-0.01047$

$0.78947-0.00838$

$0.84211-0.00628$

$0.89474 \quad-0.00419$

$0.94737-0.00209$

$1.00000 \quad 0.00000$

! Mass per unit length $(\mathrm{kg} / \mathrm{m})$

!M BMPL

$0.00000 \quad 1447.607$

$0.02105 \quad 180.333$

$0.05263 \quad 181.672$

$0.10526 \quad 183.905$

186.138

$0.21053 \quad 188.370$

$0.26316 \quad 178.321$

$0.31579 \quad 168.271$

$0.36842 \quad 158.222$

$0.42105 \quad 148.172$

$0.47368-138.123$

$\begin{array}{ll}0.52632 & 122.896\end{array}$

$\begin{array}{ll}0.57895 & 107.669\end{array}$

$0.63158 \quad 92.442$

$0.68421 \quad 77.215$ 


$\begin{array}{ll}0.73684 & 61.988 \\ 0.78947 & 51.861 \\ 0.84211 & 41.734 \\ 0.89474 & 31.607 \\ 0.94737 & 21.480 \\ 1.00000 & 11.353\end{array}$

! EI stiffness about local Y ( $\left.\mathrm{Nm}^{\wedge} 2\right)$

!M BEIYY

$0.00000 \quad 7681.46 \mathrm{E}+06$

$0.02105 \quad 1169.87 \mathrm{E}+06$

$0.05263 \quad 1020.62 \mathrm{E}+06$

$0.10526 \quad 771.88 \mathrm{E}+06$

$0.15789 \quad 523.14 \mathrm{E}+06$

$0.21053 \quad 274.40 \mathrm{E}+06$

$0.26316 \quad 234.57 \mathrm{E}+06$

$0.31579 \quad 194.74 \mathrm{E}+06$

$0.36842-154.90 \mathrm{E}+06$

$0.42105 \quad 115.07 \mathrm{E}+06$

$0.47368 \quad 75.23 \mathrm{E}+06$

$\begin{array}{ll}0.52632 & 62.49 \mathrm{E}+06 \\ 0.57895 & 49.75 \mathrm{E}+06\end{array}$

$0.63158 \quad 37.01 \mathrm{E}+06$

$0.68421 \quad 24.27 \mathrm{E}+06$

$0.73684 \quad 11.53 \mathrm{E}+06$

$0.78947-9.27 \mathrm{E}+06$

$0.84211 \quad 7.01 \mathrm{E}+06$

$0.89474 \quad 4.75 \mathrm{E}+06$

$\begin{array}{ll}0.94737 & 2.49 \mathrm{E}+06 \\ 1.00000 & 0.23 \mathrm{E}+06\end{array}$

! EI stiffness about local Z ( $\left.\mathrm{Nm}^{\wedge} 2\right)$

!M BEIZZ

$0.00000 \quad 7681.46 \mathrm{E}+06$

$0.02105 \quad 1169.87 \mathrm{E}+06$

$0.05263 \quad 1092.28 \mathrm{E}+06$

$0.10526 \quad 962.97 \mathrm{E}+06$

$0.15789 \quad 833.66 \mathrm{E}+06$

$0.21053 \quad 704.35 \mathrm{E}+06$

$0.26316 \quad 614.65 \mathrm{E}+06$

$0.31579 \quad 524.96 \mathrm{E}+06$

$0.36842 \quad 435.26 \mathrm{E}+06$

$0.42105 \quad 345.57 \mathrm{E}+06$

$0.47368-255.87 \mathrm{E}+06$

$0.52632 \quad 217.87 \mathrm{E}+06$

$0.57895 \quad 179.86 \mathrm{E}+06$

$0.63158 \quad 141.86 \mathrm{E}+06$

$0.68421 \quad 103.85 \mathrm{E}+06$

$0.73684 \quad 65.85 \mathrm{E}+06$

$0.78947 \quad 54.25 \mathrm{E}+06$

$0.84211 \quad 42.66 \mathrm{E}+06$ 

0.89474
$31.06 \mathrm{E}+06$
$\begin{array}{rr}0.94737 & 19.47 \mathrm{E}+06 \\ 1.00000 & 7.87 \mathrm{E}+06\end{array}$

! EI cross-stiffness ( $\left.\mathrm{Nm}^{\wedge} 2\right)$

!M BEIYZ

$0.00000 \quad 0.0$

$1.00000 \quad 0.0$

! GJ Stiffness about X $\left(\mathrm{Nm}^{\wedge} 2\right)$

!M BGJ

$0.00000 \quad 2655.23 \mathrm{E}+06$

$0.02105 \quad 408.80 \mathrm{E}+06$

$0.05263 \quad 343.81 \mathrm{E}+06$

$0.10526 \quad 235.50 \mathrm{E}+06$

$0.15789 \quad 127.19 \mathrm{E}+06$

$0.21053 \quad 18.87 \mathrm{E}+06$

$0.26316 \quad 16.80 \mathrm{E}+06$

$0.31579 \quad 14.72 \mathrm{E}+06$

$\begin{array}{ll}0.36842 & 12.64 \mathrm{E}+06 \\ 0.42105 & 10.56 \mathrm{E}+06\end{array}$

$0.47368 \quad 8.48 \mathrm{E}+06$

$0.52632 \quad 7.12 \mathrm{E}+06$

$0.57895 \quad 5.76 \mathrm{E}+06$

$0.63158 \quad 4.40 \mathrm{E}+06$

$0.68421 \quad 3.04 \mathrm{E}+06$

$0.73684 \quad 1.68 \mathrm{E}+06$

$\begin{array}{ll}0.78947 & 1.38 \mathrm{E}+06 \\ 0.84211 & 1.08 \mathrm{E}+06\end{array}$

$0.89474 \quad 0.78 \mathrm{E}+06$

$0.94737 \quad 0.48 \mathrm{E}+06$

$1.00000 \quad 0.18 \mathrm{E}+06$

! EA Stiffness along $\mathrm{X}\left(\mathrm{Nm}^{\wedge} 2\right)$

!M BEA

$0.00000 \quad 17152.7 \mathrm{E}+06$

$0.02105 \quad 2640.8 \mathrm{E}+06$

$0.05263 \quad 2611.3 \mathrm{E}+06$

$0.10526 \quad 2562.1 \mathrm{E}+06$

$0.15789 \quad 2512.9 \mathrm{E}+06$

$0.21053 \quad 2463.6 \mathrm{E}+06$

$0.26316 \quad 2332.8 \mathrm{E}+06$

$0.31579 \quad 2202.0 \mathrm{E}+06$

$0.36842 \quad 2071.2 \mathrm{E}+06$

$0.42105 \quad 1940.4 \mathrm{E}+06$

$0.47368 \quad 1809.6 \mathrm{E}+06$

$0.52632 \quad 1605.3 \mathrm{E}+06$

$0.57895 \quad 1401.1 \mathrm{E}+06$

$0.63158 \quad 1196.8 \mathrm{E}+06$

$0.68421 \quad 992.6 \mathrm{E}+06$

$0.73684 \quad 788.3 \mathrm{E}+06$ 


\begin{tabular}{|c|c|}
\hline 0.78947 & $654.3 \mathrm{E}+06$ \\
\hline 0.84211 & $520.4 \mathrm{E}+06$ \\
\hline 0.89474 & $386.4 \mathrm{E}+06$ \\
\hline 0.94737 & $252.4 \mathrm{E}+06$ \\
\hline 1.00000 & $118.5 E+06$ \\
\hline $\begin{array}{l}\text { ! Radius } \\
\text { !M BKMYY }\end{array}$ & of gyration about local Y (m) \\
\hline 0.00000 & 0.6680 \\
\hline 0.02105 & 0.6680 \\
\hline 0.05263 & 0.6129 \\
\hline 0.10526 & 0.5100 \\
\hline 0.15789 & 0.3840 \\
\hline 0.21053 & 0.1934 \\
\hline 0.26316 & 0.1829 \\
\hline 0.31579 & 0.1703 \\
\hline 0.36842 & 0.1550 \\
\hline 0.42105 & 0.1354 \\
\hline 0.47368 & 0.1088 \\
\hline 0.52632 & 0.1055 \\
\hline 0.57895 & 0.1010 \\
\hline 0.63158 & 0.0948 \\
\hline 0.68421 & 0.0855 \\
\hline 0.73684 & 0.0691 \\
\hline 0.78947 & 0.0680 \\
\hline 0.84211 & 0.0666 \\
\hline 0.89474 & 0.0641 \\
\hline 0.94737 & 0.0587 \\
\hline 1.00000 & 0.0409 \\
\hline $\begin{array}{l}\text { ! Radius } \\
\text { !M BKMZZ }\end{array}$ & of gyration about local z (m) \\
\hline 0.00000 & 0.6680 \\
\hline 0.02105 & 0.6680 \\
\hline 0.05263 & 0.6641 \\
\hline 0.10526 & 0.6575 \\
\hline 0.15789 & 0.6510 \\
\hline 0.21053 & 0.6446 \\
\hline 0.26316 & 0.6189 \\
\hline 0.31579 & 0.5887 \\
\hline 0.36842 & 0.5228 \\
\hline 0.42105 & 0.5089 \\
\hline 0.47368 & 0.4533 \\
\hline 0.52632 & 0.4424 \\
\hline 0.57895 & 0.4279 \\
\hline 0.63158 & 0.4079 \\
\hline 0.68421 & 0.3781 \\
\hline 0.73684 & 0.3289 \\
\hline 0.78947 & 0.3260 \\
\hline 0.84211 & 0.3218 \\
\hline 0.89474 & 0.3147 \\
\hline
\end{tabular}




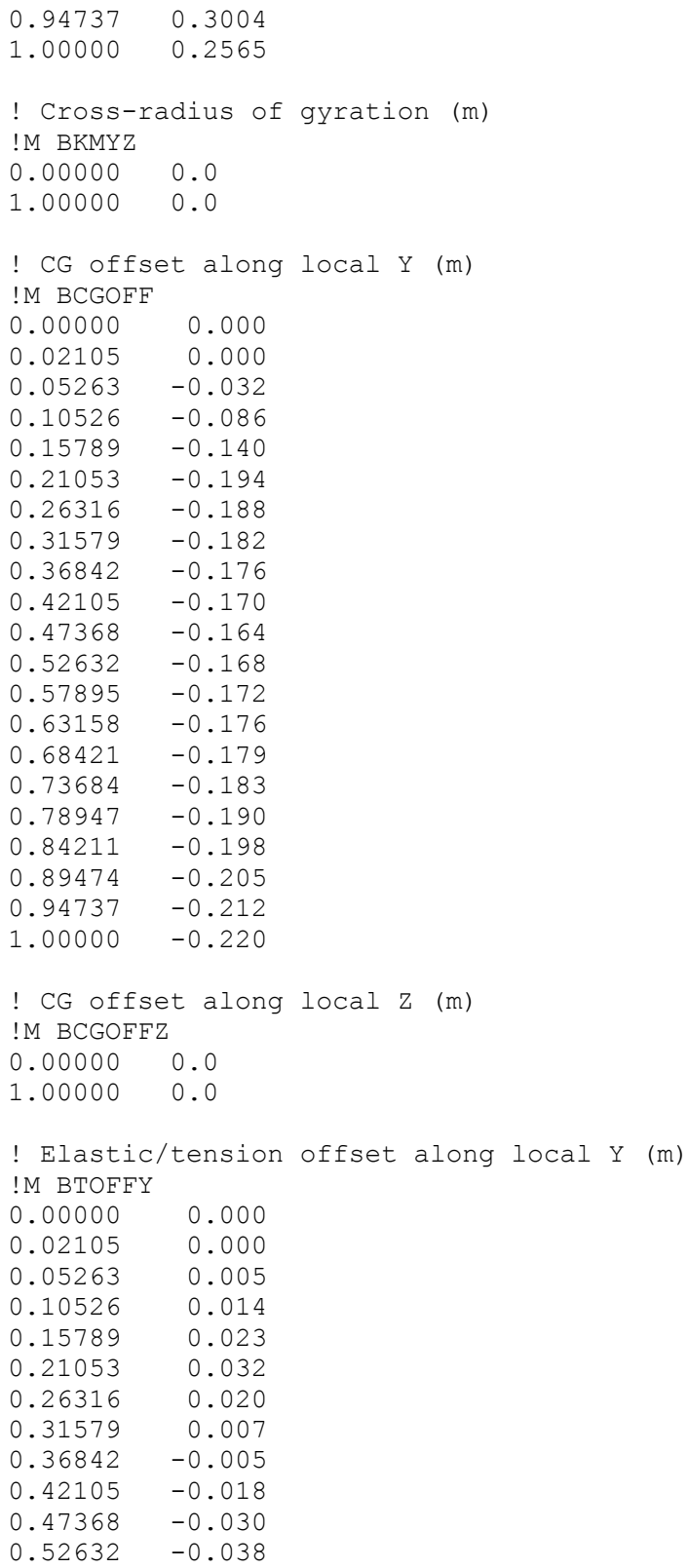




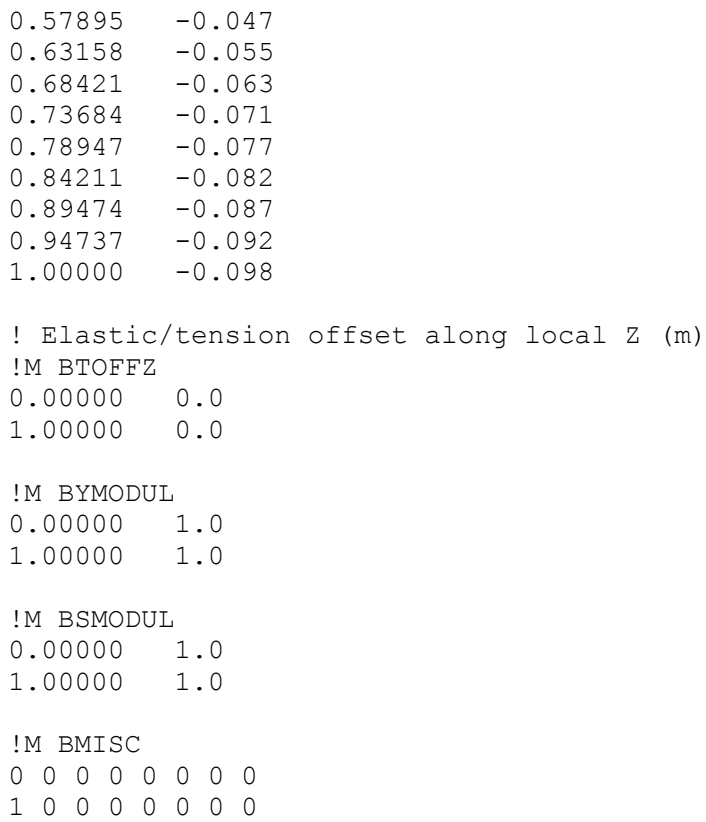




\section{RCAS Tower Properties Input File}

! 1.5 MW baseline tower model properties from "InputData1.5A08V07adm.xls" (from C. Hansen) with bugs removed.

NOTE: "! CatName" is a keyword telling RCAS that the next set of data to be read in is data for category "CatName".

Thus, the order of fields in this file is unimportant.

Reference/flexible length of beam (m)

!M REFLENGTH

82.39

! Structural twist about X (rad)

!M BSTRUCTW

$0.00000 \quad 0.0$

1.00000

! Mass per unit length $(\mathrm{kg} / \mathrm{m})$

!M BMPI

$0.00000 \quad 2549.742$

$0.11111 \quad 2275.820$

$0.22222 \quad 2017.460$

$0.33333-1774.662$

0.44444

$0.55556-1335.750$

$0.66667-1139.637$

$0.77778 \quad 959.085$

$0.88889 \quad 794.095$

$1.00000 \quad 644.666$

! EI stiffness about local Y (Nm^2)

!M BEIYY

$0.00000 \quad 243.058 \mathrm{E}+09$

$0.11111 \quad 193.660 \mathrm{E}+09$

$0.22222152 .204 \mathrm{E}+09$

$0.33333117 .790 \mathrm{E}+09$

$0.44444 \quad 89.570 \mathrm{E}+09$

$0.55556 \quad 66.753 \mathrm{E}+09$

$0.66667 \quad 48.601 \mathrm{E}+09$

$34.430 \mathrm{E}+09$

$0.88889 \quad 23.610 \mathrm{E}+09$

$1.00000 \quad 15.566 \mathrm{E}+09$

EI stiffness about local Z (Nm^2)

!M BEIZZ

$0.00000 \quad 243.058 \mathrm{E}+09$

$0.11111 \quad 193.660 \mathrm{E}+09$

$0.22222 \quad 152.204 \mathrm{E}+09$

$0.33333-117.790 \mathrm{E}+09$

$0.44444 \quad 89.570 \mathrm{E}+09$

$0.55556 \quad 66.753 \mathrm{E}+09$

$0.66667 \quad 48.601 \mathrm{E}+09$

$0.77778 \quad 34.430 \mathrm{E}+09$ 

0.88889
$23.610 \mathrm{E}+09$
1.00000
15.566

! EI cross-stiffness ( $\left.\mathrm{Nm}^{\wedge} 2\right)$

!M BEIYZ

0.0000

0.0
0.0

! GJ Stiffness about $\mathrm{X}\left(\mathrm{Nm}^{\wedge} 2\right)$

! M BGJ

$0.00000 \quad 186.968 \mathrm{E}+09$

$0.11111 \quad 148.969 \mathrm{E}+09$

$0.22222117 .080 \mathrm{E}+09$

$0.33333 \quad 90.608 \mathrm{E}+09$

$0.44444 \quad 68.900 \mathrm{E}+09$

$0.55556 \quad 51.349 \mathrm{E}+09$

$0.66667 \quad 37.386 \mathrm{E}+09$

$0.77778 \quad 26.485 \mathrm{E}+09$

$18.162 \mathrm{E}+09$

$1.00000 \quad 11.974 \mathrm{E}+09$

! EA Stiffness along $X\left(\mathrm{Nm}^{\wedge} 2\right)$

!M BEA

$0.00000 \quad 61.868 \mathrm{E}+09$

$0.11111 \quad 55.222 \mathrm{E}+09$

$0.22222 \quad 48.953 \mathrm{E}+09$

$0.33333 \quad 43.061 \mathrm{E}+09$

$0.44444 \quad 37.548 \mathrm{E}+09$

$\begin{array}{ll}0.55556 & 32.411 \mathrm{E}+09 \\ 0.66667 & 27.653 \mathrm{E}+09\end{array}$

$0.77778 \quad 23.272 \mathrm{E}+09$

$0.88889 \quad 19.268 \mathrm{E}+09$

$1.00000 \quad 15.643 \mathrm{E}+09$

! Radius of gyration about local Y (m)

!M BKMYY

$0.00000 \quad 1.9343$

$0.11111 \quad 1.8276$

0.222221 .7208

$0.33333 \quad 1.6140$

$0.44444 \quad 1.5073$

$0.55556 \quad 1.4005$

$0.66667 \quad 1.2938$

$0.77778-1.1870$

$\begin{array}{ll}0.77778 & 1.1870 \\ 1.00000 & 0.9735\end{array}$

! Radius of gyration about local Z (m)

!M BKMZZ

$0.00000 \quad 1.9343$

$0.11111=1.8276$ 


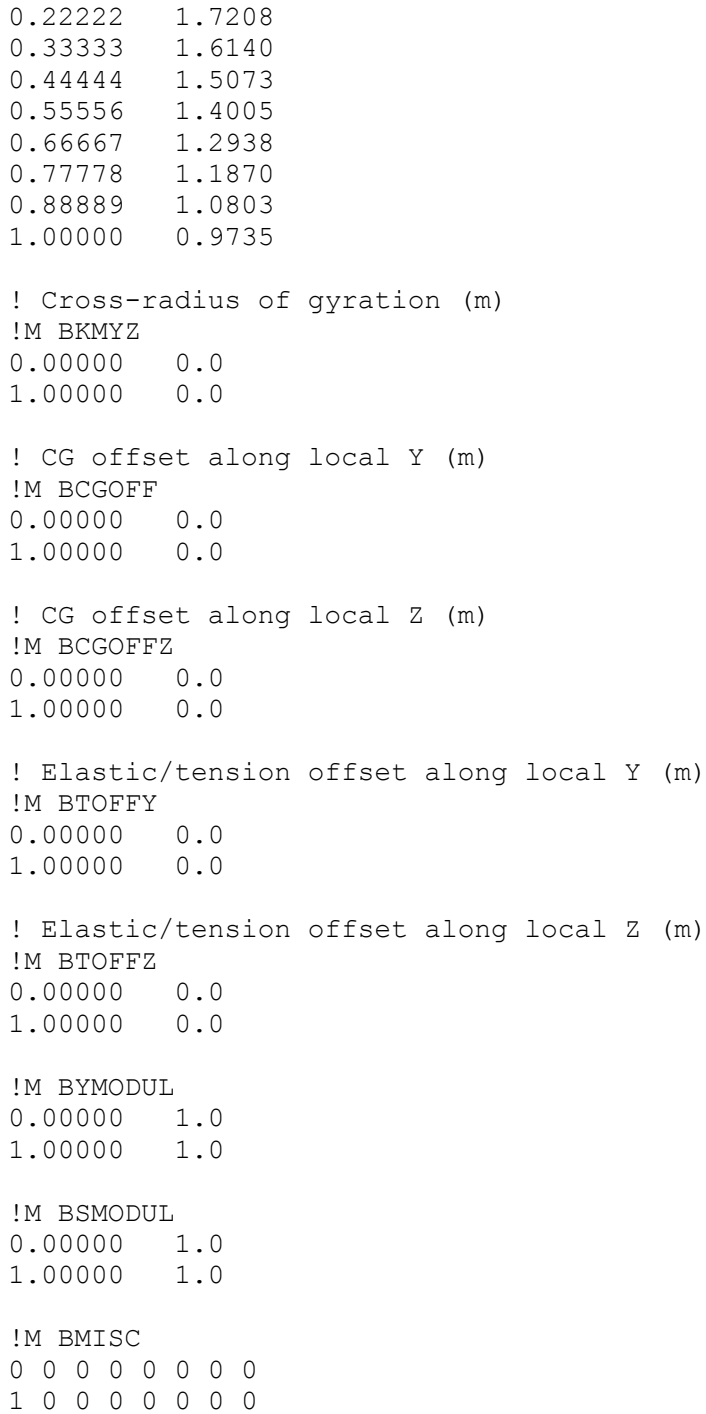




\section{RCAS Output Data Extractor File}

// This file extracts out useful information (loads and motions) from a maneuver, analysis event for the 15mwbaseline.scr models.

// Inform users we have arrived here:

$\operatorname{DISP}(" \mathrm{"})$;

DISP("Running user-defined RSCOPE script, '15mwbaselineoutputs.exc' written by J. Jonkman")

$\operatorname{DISP}(" \mathrm{n})$;

// Define some constants:

$\begin{array}{lll}\mathrm{Pi} & =3.1415927 ; & / / \mathrm{pi} \\ \mathrm{R} 2 \mathrm{D} & =57.295780 ; & / / \text { conversion from radians to degrees } \\ \text { RPS2RPM } & =9.5492966 ; & / / \text { conversion form rad/sec to rpm }\end{array}$

RPS2RPM $=9.5492966$

// conversion from radians to degrees
// conversion form rad/sec to $\mathrm{rpm}$

// Define some basic variables used for data extraction:

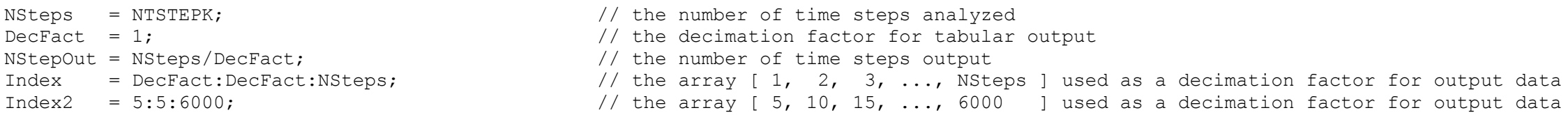

// Extract out useful loads and motions from ambiguous arrays (take data only every 5th time step):

// NOTE: the indices of these arrays where found using the DISP(@xxx) command in RSCOPE

Time

$=\operatorname{TIMEVEC}($ Index $)$

WindVxt

HorWndDir

$=$ ZEROS (NStepOut, 1$)$

$=$ ZEROS (NStepOut, 1$)$;

Node500Pos = OUTNDRPOS ( 88: 90, Index)';

global CS

Node4030XFM = OUTNDRXFM( $280: 288$, Index $)^{\prime}$;

and

BldPitch1 = UTOT $($ Index2,1);
Node400FrC $=$ OUTINTLFRC $(106: 108$, Index) ';

// extract out only every 5 th component of time

// horizontal wind speed, $\mathrm{m} / \mathrm{s}$

// wind direction, deg

// 3 components $(x, y, z)$ of position of node 500 ( deflected blade 1 tip) in

// 3 components $(x, y, z)$ of position of node 4030 (undeflected blade 1 tip) in

// 9 components of the transformation matrix of node 4030 relative to the global CS as follows:

columns 1:3 of Node4030XFM represent the 1st column of [Tp/i] of node 4030, columns 4:6 of Node4030XFM represent the 2nd column of [Tp/i] of node 4030,

// columns 7:9 of Node4030XFM represent the 3rd column of [Tp/i] of node 4030

// pitch angle of blade 1 (in degrees)

// NOTE: UTOT $=$ <values from SCREEN INITCOND> + MANEUHIS

// 3 components $(x, y, z)$ of force on node 400 (blade 1 root-blade side) in local 
Node400Mom = OUTINTLMOM(106:108, Index) ',

Node2130XFM $=$ OUTNDRXFM $(136: 144$, Index $) ' ;$

and

Node $30200 \mathrm{mg}=$ OUTNDROMG (

54, Index) ' local

Node21300mg $=$ OUTNDROMG ( axes;

48, Index)'

Node3020Frc = OUTINTLFRC ( 52: 54, Index)'; local

Node4000Mom = OUtINTLMom $(61: 63$, Index $)$ '; element CS

Node2010XFM $=$ OUTNDRXFM $(64: 72$, Index $) '$

and

Node1010XFM = OUTNDRXFM( 100:108, Index $)^{\prime}$;

and

Node1010Pos $=$ OUTNDRPoS $(34: 36$, Index $) '$ CS

Node1030Pos = OUTNDRPos $(7: 9 \text {, Index })^{\prime}$;

Node1010FrC $=$ OUTINTLFRC $(34: 36$, Index $) ' ;$ Node1010Mom = OUTINTLMOM $(34: 36$, Index $) '$,

Node1400FrC $=$ OUTINTLFRC $(40: 42$, Index $) '$; Node1400Mom $=$ OUTINTLMOM $(40: 42$, Index $) '$; element CS

/ 3 components $(x, y, z)$ of moment on node 400 (blade 1 root-blade side) in local element CS

// 9 components of the transformation matrix of node 2130 relative to the global CS as follows:

columns 1:3 of Node2130XFM represent the 1st column of [Tp/i] of node 2130, columns 4:6 of Node2130XFM represent the 2nd column of [Tp/i] of node 2130,

// columns 7:9 of Node2130XFM represent the 3rd column of [Tp/i] of node 2130

$/ / \mathrm{z}$-component of angular velocity of node 3020 (rotor end of LSS compliance) in

// element CS

$/ / \mathrm{z}$-component of angular velocity of node 2130 (intersection of yaw and shaft

// fixed in nacalle) in local element CS

// 3 components $(\mathrm{x}, \mathrm{y}, \mathrm{z})$ of force on node 3020 (rotor end of LSS compliance) in

// element CS

$/ / 3$ components $(x, y, z)$ of moment on node 4000 (rotor / hub center) in local

// 9 components of the transformation matrix of node 2010 relative to the global CS as follows:

columns 1:3 of Node2010XFM represent the 1st column of [Tp/i] of node 2010, columns 4:6 of Node2010XFM represent the 2nd column of [Tp/i] of node 2010,

columns 7:9 of Node2010XFM represent the 3rd column of [Tp/i] of node 2010

//9 components of the transformation matrix of node 1010 relative to the global CS as follows:

columns 1:3 of Node1010XFM represent the 1st column of [Tp/i] of node 1010, columns 4:6 of Node1010XFM represent the 2nd column of [Tp/i] of node 1010,

// columns 7:9 of Nodel010XFM represent the 3rd column of [Tp/i] of node 1010

// 3 components $(x, y, z)$ of position of node 1010 ( deflected tower-top) in global

// 3 components $(x, y, z)$ of position of node 1030 (undeflected tower-top) in global

// 3 components $(x, y, z)$ of force on node 1010 (yaw bearing) in local element CS // 3 components $(x, y, z)$ of moment on node 1010 (yaw bearing) in local element CS

// 3 components $(x, y, z)$ of force on node 1400 (tower base) in local element CS $/ / 3$ components $(x, y, z)$ of moment on node 1400 (tower base) in local element CS

// Calculate the blade 1 tip deflection from the deflected and undeflected tip position:

Node500Defl $=$ Node500Pos - Node4030Pos

// 3 components $(x, y, z)$ of the deflection of node 500 (blade 1 tip) in global CS 
FOR $i=1:$ NStepOut

T4030piT $(1,1: 3)=\operatorname{Node4030XFM}(i, 1: 3) ;$

T4030piT $(2,1: 3)=\operatorname{Node} 4030 X F M(i, 4: 6) ;$

T4030piT $(3,1: 3)=\operatorname{Node} 4030 X F M(i, 7: 9) ;$ END

// Calculate the rotor azimuth angle from the transformation matrices of nodes 4030 and 2130:

FOR $i=1$ : NStepOut

T4030piT $(1,1: 3)^{\prime}=\operatorname{Node} 4030 \operatorname{XFM}(i, 1: 3) ;$

T4030piT $(2,1: 3)=\operatorname{Node} 4030 X F M(i, 4: 6)$;

T4030piT $(3,1: 3)=\operatorname{Node} 4030 X F M(i, 7: 9)$;

T2130piT $(1,1: 3)=\operatorname{Node} 2130 X F M(i, 1: 3)$;

T2130piT $(2,1: 3)=\operatorname{Node} 2130 X F M(i, 4: 6)$;

T2130piT $(3,1: 3)=\operatorname{Node} 2130 \operatorname{XrM}(i, 7: 9) ;$

T4030p2130p $=$ T4030piT'*T2130piT; Azimuth $(i, 1)=\operatorname{ACOS}(\operatorname{T4} 030 p 2130 p(1,1))$;

IF T4030p2130p $(2,1)>0.0$ END

Azimuth $(i, 1)=2.0 * \mathrm{Pi}-\operatorname{Azimuth}(i, 1)$

// the transpose of transformation matrix [Tp/i] of node 4030

// 3 components $(x, y, z)$ of the deflection of node 500 (blade 1 tip) in bladelps CS END

\section{$1 /$}

$$
1 /
$$

// the transpose of transformation matrix [Tp/i] of node 2130

$$
\text { / } /
$$

// the transformation matrix [Ta/b] of node 4130 (a) relative to node 2130 (b) // use the inverse cos to obtain the rotor azimuth

$/ /$ force rotor azimuth to belong in $0<=$ azimuth $<360 \mathrm{deg}$

// Calculate the rotor speed (relative to the nacalle, not the ground):

RotSpeed = Node30200mg - Node21300mg; // rotor speed (LSS spd) relative to nacalle, not absolute

// Calculate the nacelle yaw angle from the transformation matrices of nodes 2010 and 1010:

FOR $i=1:$ NStepOut,

T2010piT $(1,1: 3)^{\prime}=\operatorname{Node} 2010 \operatorname{XFM}(i, 1: 3)$

T2010piT $(2,1: 3)=\operatorname{Node} 2010 \operatorname{XFM}(i, 4: 6)$

T2010piT $(3,1: 3)=\operatorname{Node} 2010 \operatorname{XrM}(i, 7: 9) ;$

T1010piT $(1,1: 3)=$ Node1010XFM $(i, 1: 3) ;$

T1010piT $(2,1: 3)=\operatorname{Node1010XFM}(i, 4: 6)$;

T1010piT(3,1:3) = Node1010XFM(i,7:9);

T2010p1010p = T2010piT'*T1010piT;

END

NacYaw $(i, 1)=\operatorname{REAL}(\operatorname{ASIN}(T 2010 \mathrm{p} 1010 p(2,3)))$;

$/ /$ the transpose of transformation matrix [Tp/i] of node 2010
$/ / /$
$/ / /$ the transpose of transformation matrix [Tp/i] of node 1010
$/ /$ t

// the transformation matrix [Ta/b] of node 2010 (a) relative to node 1010 (b) // use the inverse SIN to obtain the nacelle yaw angle

// Calculate the tower-top deflection from the deflected and undeflected tower-top position:

Node1010Defl $=$ Node1010Pos - Node1030Pos;

// 3 components $(x, y, z)$ of the deflection of node 1010 (yaw bearing) in global CS

// Convert the outputs to IEC-style coordinate systems: 
BladelpsCS2ConediCS $=[0.0,0.0,1.0 ; 0.0,-1.0,0.0 ; 1.0,0.0,0.0]$; // transformation matrix to convert from bladelps CS to the coned

// CS of blade

// blade 1 CS

LSShftpsCS2AzimuthCS $=[0.0,0.01 .0 ; 0.0,-1.0,0.0 ; 1.0,0.0,0.0]$; // transformation matrix to convert from LSShftps CS to azimuth CS

Node4000CS2AzimuthCS $=[-1.0,0.0,0.0 ; 0.0,-1.0,0.0 ; 0.0,0.0,1.0]$; // transformation matrix to convert from local node 4000 CS to

GlobalCS2TwrBaseCS $=[1.0,0.0,0.0 ; 0.0,-1.0,0.0 ; 0.0,0.0,-1.0]$; // transformation matrix to convert from global CS to

// tower-base CS

// tower-top / base-plate CS

TowerpsCS2TwrBaseCS $=[0.0,0.0,1.0 ; 0.0,-1.0,0.0 ; 1.0,0.0,0.0]$; // transformation matrix to convert from towerps CS to

// tower-base CS

TipDefl1 $=$ Node500Defl 1 Blade1psCS2Coned1CS

RootFb1 = Node400FrC*Node400CS2Blade1CS

RootMb1 $=$ Node400Mom*Node400CS2Blade1CS;

LSShftF $=$ Node3020FrC*LSShftpsCS2AzimuthCS

instead

effects

the

LSSTipM $=$ Node4000Mom*Node4000CS2AzimuthCS

TTDsp = Node1010Defl*GlobalCS2TwrBaseCS

YawBrF = Node1010FrC*Node1010CS2TwrTopCS

YawBrM = Node1010Mom*Node1010CS2TwrTopCS

TwrBsF = Node1400FrC*TowerpsCS2TwrBaseCS;

TwrBsM = Node1400Mom*TowerpsCS2TwrBaseCs;

// Split these into single column arrays with one array per output channel and covert units as appropriate:
/ blade 1 tip deflections in the coned CS for blade 1

$/ /$ blade 1 root forces in blade 1 CS

// root moments in blade 1 CS

// rotor thrust and rotating LSS shear forces - NOTE: use the force on node 3020

// of the force on node 4000, since the force on node 4000 doesn't include the

I/ of the hub mass (I can use the force on node 3020 to represent the forces on

// shaft tip since the thrust and shear forces are constant along the shaft).

// rotor torque and rotating LSS bending moments at the shaft tip

// tower-top deflection

$/ /$ tower-top / yaw bearing axial and shear forces

// tower-top / yaw bearing roll, pitch, and yaw moments

// tower base axial and shear forces

// tower base roll, pitch, and yaw moments

$\begin{array}{ll}\text { OoPDefl1 } & =\operatorname{TipDefl} 1(:, 1) ; \\ \text { IPDefl1 } & =\operatorname{TipDefl} 1(:, 2) ; \\ \text { RootFxb1 } & =\operatorname{RootFb1}(:, 1) / 1000.0 ; \\ \text { RootFyb1 } & =\text { RootFb1 }(:, 2) / 1000.0 ; \\ \text { RootFzb1 } & =\text { RootFb1 }(:, 3) / 1000.0 ; \\ \text { RootMxb1 } & \text { RootMb1 }(:, 1) / 1000.0 ; \\ \text { RootMyb1 } & =\text { RootMbl }(:, 2) / 1000.0 ; \\ \text { RootMzb1 } & =\text { RootMb1 }(:, 3) / 1000.0 ; \\ \text { Azimuth } & =\text { Azimuth*R2D; } \\ \text { RotSpeed } & =\text { RotSpeed*RPS2RPM; } \\ \text { RotThrust } & =\text { ISShftF }(:, 1) / 1000.0\end{array}$

RotThrust $=\operatorname{LSShftF}(:, 1) / 1000.0$; 
LSShftFya $=\operatorname{LSShftF}(:, 2) / 1000.0$; $\operatorname{LSShftFza}=\operatorname{LSShftF}(:, 3) / 1000.0$ RotTorg $=\operatorname{LSSTipM}(:, 1) / 1000.0$ LSSTipMya $=\operatorname{LSSTipM}(:, 2) / 1000.0$ LSSTipMza $=\operatorname{LSSTipM}(:, 3) / 1000.0$;

NacYaw $=$ NacYaw*R2D

TTDspFA $=\operatorname{TTDsp}(:, 1)$;

TTDspSS $=\operatorname{TTDsp}(:, 2)$;

YawBrFxp $=\operatorname{YawBrF}(:, 1) / 1000.0$

YawBrFyP = YawBrF $(:, 2) / 1000.0 ;$

YawBrEyP = YawBrE $(:, 2) / 1000$

YawBrMxp = YawBrM $(: 1,1) / 1000.0$

YawBrMyP = YawBrM $(: 2,2) / 1000.0$

YawBrMyp = YawBrM $(:, 2) / 1000.0$

YawBrMzp $=$ YawBrM $(:, 3) / 1000.0$

TwrBsFxt $\operatorname{TwBSF}(:, 1) / 1000.0$

TwrBsFyt $=\operatorname{TwrBSF}(:, 2) / 1000.0$

TwrBsFzt $=\operatorname{TwrBsF}(:, 3) / 1000.0$

TwrBsMxt $=\operatorname{TwrBsM}(:, 1) / 1000.0$

TwrBsMyt = TwrBsM $(: ; 2) / 1000.0 ;$

TwrBsMzt $=\operatorname{TwrBsM}(:, 3) / 1000.0$;
// y-component of rotating LSS shear force, kN

// z-component of rotating LSS shear force, kN

// rotor torque, $\mathrm{kN}-\mathrm{m}$

// y-component of rotating LSS bending moment at the shaft tip, kN-m

// z-component of rotating LSS bending moment at the shaft tip, kN-m

// nacelle yaw angle, deg

// FA tower-top deflection, m

// SS tower-top deflection, m

// $\mathrm{x}$-component of tower-top / yaw bearing shear force, kN

// y-component of tower-top / yaw bearing shear force, kN

// tower-top / yaw bearing axial force, $\mathrm{kN}$

// tower-top / yaw bearing roll moment, kN-m

// tower-top / yaw bearing pitch moment, kN-m

// tower-top / yaw bearing yaw moment, kN-m

// $\mathrm{x}$-component of tower base shear force, kN

$1 / \mathrm{y}$-component of tower base shear force, kN

// tower base axial force, $\mathrm{kN}$

// tower base roll moment, $\mathrm{kN}-\mathrm{m}$

// tower base pitch moment, $\mathrm{kN}-\mathrm{m}$

// tower base yaw moment, kN-m

// Write the data to file "15mwbaseline.out":

SHORTE;

// use short, exponential format for outputs

PRINT( "15mwbaseline.out", Time, WindVxt, HorWndDir, OoPDefl1, IPDefl1, BldPitch1, RootFxb1, RootFyb1, RootFzb1, RootMxb1, RootMyb1, RootMzb1, Azimuth, RotSpeed, RotThrust, LSShftFya, LSShftFza, RotTorq, LSSTipMya, LSSTipMza, NacYaw, TTDspFA, TTDspSS, YawBrFxp, YawBrfyp, YawBrfzp, YawBrMxp, YawBrMyp, YawBrMzp, TwrBsfxt, TwrBsfyt, TwrBsfzt, TwrBsMxt, TwrBsMyt, TwrBsMzt ); 


\section{REPORT DOCUMENTATION PAGE}

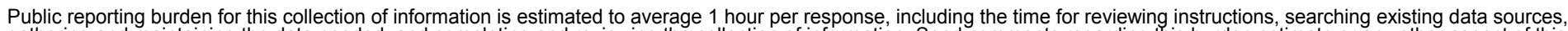

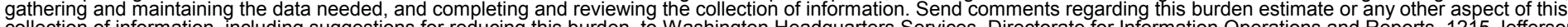
Davis Highway, Suite 1204, Arlington, VA 22202-4302, and to the Office of Management and Budget, Paperwork Reduction Project (0704-0188), Washington, DC 20503.
1. AGENCY USE ONLY (Leave blank)
2. REPORT DATE
August 2003

\section{REPORT TYPE AND DATES COVERED}
Technical Report

4. TITLE AND SUBTITLE

A Demonstration of the Ability of RCAS to Model Wind Turbines

6. AUTHOR(S)

J. Jonkman and J. Cotrell

7. PERFORMING ORGANIZATION NAME(S) AND ADDRESS(ES)

National Renewable Energy Laboratory

1617 Cole Blvd.

Golden, CO 80401-3393

9. SPONSORING/MONITORING AGENCY NAME(S) AND ADDRESS(ES)
5. FUNDING NUMBERS

WER3.2050

8. PERFORMING ORGANIZATION REPORT NUMBER

10. SPONSORING/MONITORING AGENCY REPORT NUMBER

NREL/TP-500-34632

11. SUPPLEMENTARY NOTES

12a. DISTRIBUTION/AVAILABILITY STATEMENT

National Technical Information Service

U.S. Department of Commerce

5285 Port Royal Road

Springfield, VA 22161

13. ABSTRACT (Maximum 200 words)

In recent years, the wind industry has sponsored the development, verification, and validation of comprehensive aeroelastic simulators, which are used for wind turbine design, certification, and research. Unfortunately, as wind turbines continue to grow in size and sometimes exhibit unconventional design characteristics, the existing codes do not always support the additional analysis features required for proper design. The development history, functionality, and advanced nature of RCAS (Rotorcraft Comprehensive Analysis System) make this code a sensible option. RCAS is an aeroelastic simulator developed over a 4-year cooperative effort amongst the U.S. Army's Aeroflightdynamics Directorate, Advanced Rotorcraft Technology (ART), Inc., and the helicopter industry. As its name suggests, RCAS was created for the rotorcraft industry but developed as a general purpose code for modeling the aerodynamic and structural response of any system with rotating and nonrotating subsystems (such as wind turbines). To demonstrate that RCAS can analyze wind turbines, models of a conventional, 1.5-MW, 3-bladed, upwind, horizontal axis wind turbine (HAWT) are created in RCAS and wind turbine analysis codes FAST (Fatigue, Aerodynamics, Structures, and Turbulence) and ADAMS (Automatic Dynamic Analysis of Mechanical Systems). Using these models, a side-by-side comparison of structural response predictions is performed under several test scenarios.

wind energy; FAST; ADAMS; wind turbine; areoelastic simulators; RCAS; modeling

15. NUMBER OF PAGES

16. PRICE CODE

17. SECURITY CLASSIFICATION OF REPORT

Unclassified
18. SECURITY CLASSIFICATION OF THIS PAGE Unclassified
19. SECURITY CLASSIFICATION OF ABSTRACT

Unclassified
20. LIMITATION OF ABSTRACT

UL 